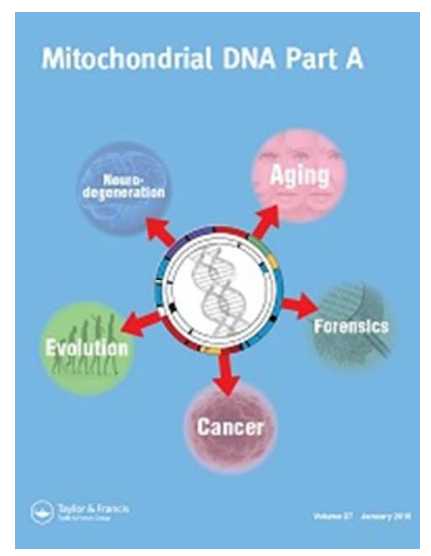

Deep sequencing reveals the mitochondrial DNA variation landscapes of breast-to-brain metastasis blood samples

\begin{tabular}{|r|l|}
\hline Journal: & Mitochondrial DNA Part A \\
\hline Manuscript ID & GDNA-FLRP-2017-0109 \\
\hline Manuscript Type: & Full length Research Paper \\
\hline Date Submitted by the Author: & 18 -Apr-2017 \\
\hline Complete List of Authors: & $\begin{array}{l}\text { McGeehan, Rhiannon; University of Portsmouth, School of Pharmacy and } \\
\text { Biomedicine } \\
\text { Cockram, Lewis; University of Portsmouth, Institute of Biomedical and } \\
\text { Biomolecular Science } \\
\text { Keatley, Kathleen; University of Portsmouth, Institute of Biomedical and } \\
\text { Biomolecular Science } \\
\text { Eccles, Diana; University of Southampton, Faculty of Medicine } \\
\text { An, Qian; University of Portsmouth, School of Pharmacy and Biomedicine }\end{array}$ \\
\hline Keywords: & $\begin{array}{l}\text { mtDNA, breast to brain metastasis, 3D protein structural mapping and } \\
\text { analysis, breast cancer }\end{array}$ \\
\hline
\end{tabular}

SCHOLARONE $^{\text {m }}$

Manuscripts 


\title{
Deep sequencing reveals the mitochondrial DNA variation landscapes of breast-to-brain metastasis blood samples
}

\author{
Rhiannon E. McGeehan ${ }^{1 *}$, Lewis A. Cockram ${ }^{1,2 \$}$, Kathleen Keatley ${ }^{1,3}$, Diana M. Eccles ${ }^{4}$ and Qian \\ $\mathrm{An}^{2 *}$ \\ 1 Brain Tumour Research Centre, Institute of Biomedical and Biomolecular Sciences, University of \\ Portsmouth, Portsmouth, PO1 2DT, UK \\ 2 School of Pharmacy and Biomedical Sciences, Institute of Biomedical and Biomolecular Sciences, University \\ of Portsmouth, Portsmouth, PO1 2DT, UK \\ ${ }^{3}$ School of Biological Sciences, Institute of Biomedical and Biomolecular Sciences, University of Portsmouth, \\ Portsmouth, PO1 2DT, UK \\ 4 Cancer Sciences Academic Unit, Faculty of Medicine, University of Southampton, Somers Cancer Research \\ Building MP824, Tremona Road, Southampton, SO16 6YA, UK \\ $\$$ Joint first authors \\ * Correspondence: rhiannon.mcgeehan@port.ac.uk or qian.an@port.ac.uk; Tel.: +44 2392842556
}

\begin{abstract}
Breast-to-brain metastasis (BBM) often represents a terminal event, due to the inability of many systemic treatments to cross the blood-brain barrier (BBB), rendering the brain a sanctuary site for tumour cells. Identifying genetic variations that can predict the patients who will develop BBM would allow targeting of adjuvant treatments to reduce risk while disease bulk is minimal. Germ-line genetic variations may contribute to whether a BBM forms by influencing the primary tumour sub-type that presents, or by influencing the host-response to the tumour or treatment regimen, or by facilitating transition of tumour cells across the BBB and establish a viable brain metastasis. The role of mitochondrial DNA (mtDNA) variants specifically in BBM is underexplored. Consequently, using a sensitive deep-sequencing approach, we characterised the mtDNA variation landscapes of blood samples derived from 13 females who were diagnosed with early onset breast cancer and later went on to develop BBM. We also predicted the potential pathogenic significance of variations identified in all mtDNA-encoded oxidative phosphorylation (OXPHOS) proteins using 3D protein structural mapping and analysis, to identify variations worthy of follow-up. From the 70 variations found in protein coding regions, we reveal novel links between 3 specific mtDNA variations and altered OXPHOS structure and function in $23 \%$ of the BBM samples. Further studies are required to confirm the origin of mtDNA variations, whether they correlate with 1) the predicted alterations in mitochondrial function and 2) increased risk of developing breast brain metastasis using a much larger cohort of samples.
\end{abstract}

Keywords: mtDNA; breast cancer; breast to brain metastasis; 3D protein structural mapping and analysis; OXPHOS and long PCR

\section{Introduction}

Breast neoplasms are currently the second highest cause of cancer related death in women. Although brain metastases are less common than lesions in the bone or lungs, they often represent a terminal event with a projected survival of just 3 to 6 months from diagnosis, with less than $20 \%$ of patients surviving more than 1 year [1-3]. Poor clinical outcome is due, in part, to the difficulty in treating the metastatic site, with systemic treatments generally being unable to penetrate the blood brain barrier. Predicting clinical outcome is also difficult due to the complicated interactions of metastatic cells with host homeostatic mechanisms, which metastatic cells ultimately exploit for their own survival and proliferation [4].

The process of brain metastasis, including breast-to-brain metastasis (BBM), consists of a series of steps. In order that a clinically relevant brain lesion is observed, tumour cells must: 1) reach $>1 \mathrm{~mm}^{3}$ by rapidly proliferating and establishing new vasculature, 2 ) invade the host cells and gain access to either the circulatory lymphatic or haematogenous systems, 3) survive the lymphatic or 
haematogenous systems and arrest in the capillary beds and extravasate into the brain, and 4) proliferate and form metastasis in the brain [4]. Despite this broad understanding of the metastatic process, the incidence of BBM is on the increase and there is consequently a need for better understanding of the BBM process as well as markers that could predict which patients will develop BBM, which in turn could improve current treatments for patients with BBM.

Patient-derived blood samples are often used to identify associations between genetic variations in the germ-line and cancer prognosis $[5,6]$. Identifying such variations could help predict which patients may develop BBM, allowing the targeting of adjuvant treatments aimed at reducing risk while the disease bulk is minimal.

MtDNA, which exists as multiple copies in the mitochondrial matrix, is 16,569 base pairs long and encodes 37 genes. These genes provide 13 key catalytic proteins of the oxidative phosphorylation (OXPHOS) system, as well as the 2 rRNAs (12S and 16S) and 22 tRNAs vital for their synthesis. Of the $13 \mathrm{mtDNA}$-encoded proteins, 7 contribute to complex I (NADH dehydrogenase), 1 contributes to complex III (cytochrome bc1 complex), 3 contribute to complex IV (cytochrome c oxidase) and 2 contribute to complex V (ATP synthase).

The OXPHOS system is composed of the mitochondrial respiratory chain (complexes I to IV) and ATP synthase (complex V). This system is vital for the production of cellular energy in the form of ATP and also has numerous secondary roles in other processes including calcium homeostasis [7], reactive oxygen species (ROS) production and signaling [8] and apoptosis $[9,10]$. Due to the importance of OXPHOS, variations in the mtDNA sequence, which can be germ-line or somatic, have the capacity to inflict profound effects on cellular function, contributing to or causing numerous human diseases (reviewed in [11]). As several hundred mtDNA molecules co-exist within a cell or tissue however, each with the capacity to hold different variations, a certain threshold must be met for a variation toexert an observable phenotypic effect [12]. The occurrence of multiple mtDNAs within a population is termed heteroplasmy.

Alterations in OXPHOS, as a consequence of mtDNA variations, have a proposed role in metastasis [13]. For example, transmitochondrial hybrids (cybrids), formed through the cell-cell fusion of enucleate highly metastatic lung cancer cells and mtDNA deplete lung cancer cells with low metastatic potential, enhanced the recipient cell's ability to form metastatic nodules in the lungs of mice following tail vein inoculation [14]. Consistent with this, the reciprocal mtDNA exchange suppressed the ability of the host cells to form metastatic nodules [14]. Despite the various studies that have looked into the incidence and predisposing roleof mtDNA variations in primary breast cancer [15-18] and the role of mtDNA variations in the metastatic process using in vitro cellular and mouse xenograft models [13-14, 46], no similar studies have been conducted in the context of BBM.

As a first step towards determining whether mtDNA variations in the germ-line could influence the likelihood of developing breast to brain metastasis, in this study we determined the blood mtDNA variation landscapes of 13 breast to brain metastasis patients using an established, sensitive deep sequencing approach [19]. As expected, numerous mtDNA variations were detected relative to the revised Cambridge reference sequence (rCRS [23] [Genbank: NC_012920]). Consequently, in order to draw up a short-list of candidates worthy of follow up investiagtion, all of the non synonymous variations identified in mtDNA-encoded OXPHOS proteins were then inspected in detail in silico using the latest, best quality and homologous 3D protein structure data available to analyse and predict their potential functional impact on the OXPHOS system [20] and therefore potential links with the BBM process. Until now, this has only been possible for mtDNA-variations in complex III and IV genes $[19,20]$. However, recent publication of the cryo-EM $3 \mathrm{D}$ structures for complexes I and V [28,29, respectively] means, for the first time, we can also gain insight into the impact of mtDNA-variations at the level of protein structure in these complexes.

\section{Materials and Methods}

2.1 Breast to brain metastasis patients and DNA samples

URL: http://mc.manuscriptcentral.com/gdna Email: desalle@amnh.org 
Thirteen whole blood DNA samples were obtained from a subset of the Prospective study of Outcomes in Sporadic vs Hereditary breast cancer (POSH) [21,22]. Specifically, these patients had developed breast-to-brain metastasis. Patients were aged 40 or younger, and had been diagnosed with invasive breast cancer between January 2000 and January 2008. They also had varying NPI (Nottingham prognostic index) scores, hormone receptor expression and treatment exposure (Table S1). The NPI is used to calculate the prognosis of breast cancer patients following surgery, taking into consideration the size of the lesion, the number of lymph nodes affected and the tumour grade (I-III). As a collective, the patients in this study had an average NPI score of just above 5.4, meaning $70 \%$ of patients had a projected survival of 5 years.

\subsection{Long PCR amplification and sequencing of complete mtDNAs}

Based on the method of Lloyd et al. 2015 [19], total DNA was extracted from the total blood of each patient using DNeasy Blood \& Tissue Kit (Qiagen) according to the manufacturer's instructions. 100ng of total whole blood DNA of each patient, was amplified using two overlapping mtDNA specific primer pairs: CytbF and HumanLongR (Amplicon 1) or HumanLongF and CytbR (Amplicon 2) to yield two halves of the mitochondrial genome in each case, which were then resolved by agarose gel electrophoresis, purified and quantified (Table S2). Amplicon 1 and 2 from each sample were pooled ( $0.65 \mathrm{nM}$ each) and $1 \mathrm{ng}$ of DNA from each pool was used to construct 13 Truseq Nano libraries (Illumina) which were combined, sequenced with paired-end 2 X 150 reads on the Illumina MiSeq system (Department of Biochemistry, University of Cambridge).

\subsection{Identification of variations, determination of heteroplasmy and annotation}

Post sequencing, reads obtained from the Illumina Resequencing Workflow (http://supportres.illumina.com/documents/documentation/software_documentation/miseqreporter/miseq-repo rter-user-guide-15042295-b.pdf), were aligned against the rCRS [23] [Genbank: NC_012920] using the Burrows Wheeler Aligner (Galaxy Tools Version (GTv) 1.2.3). The rCRS, which represents haplogroup $\mathrm{H} 2 \mathrm{a} 2$, is currently the standard sequence used for identifying the presence of mtDNA variations. Refinement of the initial alignment was then conducted through the marking and removal of duplicate reads, using Picard beta (GTv 1.56.0), followed by setting up regions for indel realignment, realignment around indels, identification of co-variates and recalibration of quality scores using the GATK tools beta (GTv 0.0.4, 0.0.6, 0.0.5 and 0.0.6, respectively).

Variant calling was carried out on the processed alignment with the GATK unified genotyper (GTv 0.0.6) to identify regions of the sequences that diverged from the rCRS. Complete processing of reads through this workflow removed the presence of several suspected false variations (G3483C, T3488A, A3492C and 3492insC), that frequently reside in the unprocessed variant files generated directly by the Illumina Resequencing Workflow.

To further minimise miscalling of variations, those which had previously been identified as "false" variants due to misalignments around homopolymer tracts or sequencing errors by Ju et al. (A302C, C309T and C3106A; [27]) were discarded. Finally, variants at sites in the rCRS which are known to contain rare alleles $(A 263, A 750, A 1438, A 8860$ and $A 15326)$ were not considered in further analysis [23].

To confirm the presence and calculate the heteroplasmy of each of the remaining variations a minimum read quality (Phred) score of $30(\mathrm{Q} 30)$ was applied, this means that there is a very low likelihood (just a $0.01 \%$ chance) that the variations detected were due to sequencing error. To be confident that the variants called were not due to sequencing error a stringent heteroplasmy cut off of $\geq 1 \%$ was applied, i.e. the number of mtDNA molecules harboring the variant had to be $\geq 1 \%$ or $\leq 99 \%$ to be considered heteroplasmic. If $<1 \%$ then the variant would be considered homoplasmic wild-type. If $>99 \%$ then the variant would be considered homoplasmic (pure) mutant. To determine heteroplasmy, 250 of the Q30-reads (with the duplicates removed) in the alignment were chosen at random and then heteroplasmy was calculated by dividing the depth of reads with the variant nucleotide by 250 , and then expressed as a percentage. The presence of variations was further confirmed by visually inspecting the full alignments using Tablet[47]. Annotation of the identified 
variations was conducted manually by inputting variations into Mitowheel (http://mitowheel.org/mitowheel.html) to ascertain their locus and precise position in the mitochondrial genome.

\subsection{Prevalence of variations in normal/healthy (control) bloods and pathologic tissues}

Previous disease associations of the variations were established through MITOMAP (http://www.mitomap.org/MITOMAP) and the human mitochondrial database (HmtDB; http://www.hmtdb.uniba.it/hmdb/). The HmtDB was also used to determine the prevalence of variations within bloods obtained from normal, healthy individuals (as controls). The HmtDB contains over 28,196 and 3,539 mitogenomic sequences obtained from the tissues of normal, healthy subjects and patients, respectively.

\subsection{Identifying functional candidates using three-dimensional protein structure mapping and analysis}

Three-dimensional (3D) protein structure mapping and analysis was used to examine and predict the functional impact of all non synonymous variations identified in mitochondrial encoded proteins. This approach was developed and validated on a number of complex III and complex IV variations associated with a broad range of human diseases, and has more recently been used to identify mtDNA variations worthy of follow up investigations using in vitro functional assays in glioblastoma [19,20, respectively]. Subsequently, all variations found in GBM MT-CYBs and predicted to be of significance have been shown to influence complex III propertiessuch as activity or drug-sensitivity, when individually introduced into yeast, validating the usefulness of the approach [48]. The recent high profile publications of both the mammalian complex I [28] and complex V [29] cryo-EM structures, means here, for the first time the pathogenicity of mtDNA variations within these complexes can also be predicted, something which precluded the earlier studies $[19,20]$.

In brief, the 13 human mitochondrial protein sequences were used to find the latest, best quality and most similar OXPHOS complex structures for complexes I, III, IV and V available from RCSB PDB (complex I - [PDB: 5LC5] (B. taurus) [28]; complex III - [PDB:1NTZ] (B. taurus) [30] \& [PDB:1BE3] (B. taurus) [31] ; complex IV [PDB:2EIJ] (B. taurus) [32] and complex V [PDB:5ARA] (B. taurus) [29]. Similarity between human sequences and the new complex I and V models are shown in Table S3, while the others have been documented previously [20].

Relative location and conservation of variations between the human and model sequence alignments was identified using ClustalW2 [33] and then annotated with ESPript [34]. Variations were mapped to their most homologous associated structures and displayed in PyMOL (Schrödinger, LLC). In the case of complex I, maps were generated using the X-ray generated $T$. thermophilus [PDB:4HEA] model, as this was not possible using the cryo-EM generated B. taurus [PDB: 5LC5] model, which is not available with docked X-ray crystallographic data. Detailed analysis of the effect of variations, based on their proximity to important structural features within the models, was then performed on the most similar structures using COOT [35]. Following this, variations were classified into 1 of 5 distinct pathogenic categories: (i) frameshift, (ii) active site, (iii) binding pocket, (iv) protein interaction region or (v) non pathogenic.

\section{Results and Discussion}

\subsection{Generation of new complete mitochondrial genomes}

Long PCR and sequencing of whole blood DNA from BBM patients yielded 13 high quality new complete mitochondrial genomes (BBM1-13]. Each genome was compiled from 1,626,861 $\pm 196,610$ reads with a mean length of $150.2 \pm 0.3$ nucleotides (Table 1 ). These reads were then mapped against the revised Cambridge Reference Sequence (rCRS [23]), forming alignments with an average total of 
$1,559,947 \pm 196,351$ reads and a mean depth of coverage per nucleotide of $14,224 \pm 1,781$ over the entire mitochondrial genome (Table 1).

\subsection{Highly variable $m t D N A$ profiles}

In total, 330 variations were identified across the 13 mitochondrial genomes; this consisted of 160 nucleotide positions that were found to be altered in just one or multiple samples (detailed in Table S4). The vast majority of the total variations (274/330, 83\%) were nucleotide substitutions with the remaining variations being indels, including 36 insertions and 20 deletions.

The average number of variations in each sample was $25.38 \pm 15.98$, with most variations occurring in BBM4 and BBM8, which contained 58 and 53, respectively. BBM6, BBM7 and BBM11 contained just 11 variations each (Figure 1A). The range of heteroplasmy for each variation detected in each sample was also diverse, with no significant differences in the average heteroplasmy $(72.03 \%$ $\pm 34.84 \%$ ) observed between samples (Figure 1B). The total number of variations and average heteroplasmy observed in this small cohort of individual samples did not appear to correlate with markers known to be associated with the likelihood of developing BBM, i.e. triple negative or Her2+, or ER- (see Rostami et al. for review [36]), nor whether the patient had been exposed to radio- or chemotherapy prior to the blood DNA being taken for analysis (Table S1), despite some studies showing chemotherapies can result in mtDNA instability [37]. This prompted us to look in more detail at the specific variations present.

\subsection{D-loop, $m t n d 4, m t-n d 5$ and $m t$ cyb are variation hotspots}

Variations were identified in all regions of the mtDNA genome apart from $m t n d 3$ (Figure 2A). Variations were predominantly situated in the displacement loop ( $D$ loop), a non coding control region and known "hotspot" for mtDNA variation in many cancers [38,39], which contained $45 \%$ $(149 / 330)$ of all variations.

In the coding regions, the largest number of variations (12\%; 41/330) occurred in $m t r n r 2$, which encodes for the mitochondrial $16 \mathrm{~S}$ rRNA. Three other mitochondrial coding genes also contained a high number of variations: $m t n d 4$ and $m t n d 5$ (both encoding complex I subunits) each contained $5 \%$ $(18 / 330)$ and $m t c y b$ (encoding for a complex III subunit), which contained $6 \%(19 / 330)$ of the total variations. These patterns did not appear to be correlated with locus length (Figure 2B).

\subsection{Heteroplasmy, prevalence and disease-association characteristics of recurrent variations}

Seventy out of 160 variations appeared in 3 or more samples (Table S4). Thirty-two (46\%) of the recurrent variations were identified in the D-loop and included: A73G, T152C, C299A, A301C, 302insC, T310C, 310insC, A16183C, C16256T, T16311C, T16326C and T16519C. The remaining 38 $(54 \%)$ of the recurrent variations were present in the RNA-coding regions. The most frequently recorded variations were (in descending order of frequency): A2706G in $m t ~ r n r 2, C 7028 \mathrm{~T}$ in $m t$ co1, A11719G in $m t-n d 4, A 12308 G$ in $m t t l 2, C 14766 \mathrm{~T}$ in $m t c y b, A 11467 G$ in $m t n d 4, G 12372 A$ in $m t n d 5$ and G709A in $m t$ rnr1.

Broadly speaking, the recurrent variations could be categorised into two groups (Table S4):

(1) a large group of almost $100 \%$ heteroplasmy, probably reflecting a germ-line origin. Among these, three subgoups were identified. Those which were found at a similar frequency in the bloods of BBM patients and normal subjects (A2706G, C7028T, T152C, T16311C and T16362C) and therefore unlikely to be of significance, those which were enriched by $10 \%$ or more frequent in the bloods of BBM patients than normal subjects (A11467G, G12372A, G709A, A12308G and C16256T) and therefore potentially significant, and finally, those which were depleted by $\sim 10 \%$ or more in the bloods of BBM patients than normal subjects (A11719G, C14766T, A73G and T16519C).

(2) a small group with reasonably low average heteroplasmy (33.69 $\pm 29 \%$ ), which could be of either germ-line or tumour cell origin. All of the variations in this group were enriched by $\sim 10 \%$ or more in the bloods of BBM patients when compared to normal subjects (C299A, A16183C, A301C, $302 i n s C, T 310 C$ and 310ins $C$ ) and therefore of potential significance. Interestingly, among the 
somatic variations detected in primary breast cancer cells, those somatic variations detected in the "D310 repeat region of the D-loop", of which T310C and 310insC (this study) would be examples, have been promoted as marker for breast tumourigenesis [40]. C16256T has been detected in breast and brain tumours previously; T152C, A11467G, G12372A, A12308G and A16183C have been detected in multiple tumour types, including breast and brain tumours previously; T16362C, T16519C and T310C have been detected in multiple tumour types including brain tumours previously; and $A 73 G$ and $T 16311 C$ have been detected in multiple tumour types previously, although not breast or brain tumours. On the other hand, A2706G, C7028T, A11719G, C14766T, G709A, C299A, A301C, 302insC and 310insC have not been reported in tissues of patients with pathology previously (Table $\mathrm{S} 4$ ).

Although, there is evidence that suggests mtDNA variations can play a role in primary breast cancer, e.g. the germ-line variations: T16189C [15], G9055A, T16519C, T239C and C16207T [16,17] are associated with breast cancer development susceptibility and G10398A is associated with higher breast cancer risk in African women [18], these were not among the recurrent variations in our cohort.

\subsection{Global structural maps}

Overall 116 variations were identified in protein coding regions, of these $79(68 \%)$ were synonymous and $37(32 \%)$ were non synonymous (Figure 3A). All non-synonymous variations in the mtDNA-encoded OXPHOS genes were mapped onto their corresponding structural homologs, yielding the first insight into their distribution across the complexes. Figure 4, which is a compendium of the 23 non-synonymous variations identified in all of the BBM patients, reveals a non-uniform distribution. Most variations were found in (in descending order): complex I, IV, III and V. The high frequency of variations in complexes I, IV and III can be accounted for by variation hotspots (again in descending order) in MT-ND1 and ND5 of complex I, MT-CO3 of complex IV and MT-CYB of complex III, respectively. MT-ATP6 also contained a couple of variations, while MT-CO1, MT-CO2, MT-ND2 and MT-ND6 each contained just one variation; MT-ND3, ND4, ND4L, and MT-ATP8 (not shown), however, remained variation free. Among the non-synonymous variations, $48 \%$ (11 of 23 ) were conserved between the human sequences and the bovine models (Figure S1). Despite complex I containing most of the non-synonymous variations, just 30\% (3 of 10) were conserved, followed by $40 \%$ in complex III ( 2 of 5), $60 \%$ in complex IV (4 of 6) and $100 \%$ in complex V (2 of 2)(Figure 4, Figure S1 and Table S3).

\subsection{Three functional candidates identified}

Owing to the $61-91 \%$ identity that exists between the human sequences and the homologs ([19] and Table S3), we were able to perform, for the first time, detailed 3D structural analysis on all complexes of the OXPHOS system containing mtDNA-encoded subunits. Using this approach, we were able to predict just 3 out of the 23 non-synonymous variations were likely to have a functional impact at the level of protein structural changes, and could be put into previously defined structural/functional classes $[19,20]$. One occurred in a complex III ligand/inhibitor binding pocket region (class 3) and two occurred in protein-protein interaction regions (class 4), one in complex IV and one in complex $\mathrm{V}$. The remaining 9 variations, including all 10 in complex I, are predicted to be non-functional (Table 2).

The absence, or very low frequency, of all 3 of the functional candidates in the bloods of normal healthy individuals further hints that the variations could be important. According to the HmtDB: the complex IV variation (E153G) has previously been documented in diabetes, while T14819insTTCTATA and S99P have not been previously been documented were not reported (Table S4 and S5). Intriguingly, S99P has been reported as a somatic variant in the TCGAs-BRCA project of primary breast invasive carcinoma patients. 


\subsection{Mechanistic insights}

The structural consequences of the 3 variations predicted to be functional are illustrated in Figures 5 and 7.

Figure 5A to D depicts the structural consequence of the 14819insTCTATA in frame insertion on the only mtDNA-encoded subunit of Complex III: MT-CYB. Complex III is at the centre of the OXPHOS system, and catalyses the transfer of electrons from ubiquinol to cytochrome $\mathrm{c}$, which is coupled with the translocations of protons across the inner mitochondrial membrane. Complex III is also a major site for ROS production. MT-CYB lies at the centre of dimeric complex III, and forms intimate interactions with 20 to 22 additional subunits (10 to 11 per MT-CYB monomer) that are nuclear DNA-encoded. The subunit contains two hemes and two inhibitor binding sites, the Qo and Qi sites. Although the codons remain in register, the introduction of 6 new nucleotides at position 14819 of the mitochondrial genome eventually causes a serine to phenylalanine substitution at position 25 before the addition of a further 2 new amino acids, a tyrosine and a threonine at position 26 and 27, respectively, of the MT-CYB polypeptide (Figure S2). Although it is difficult to model the precise effects of these changes, such a substantial change is unlikely to be easily accommodated by the wild type protein (Figure 5A, C) and we predict local mis-folding of the mutant MT-CYB in a region proximal to the ubiquinol/ubiquinone binding site (the Qi-site; Figure $5 \mathrm{~B}$ ). The mis-folding could also affect the surface of MT-CYB (Figure 5C, D), disrupting its interactions with the nuclear subunits, and therefore the stability of complex III. Taken together, 14819insTCTATA is likely have an effect on complex III activity. As inhibitors can also bind to the Qi-site, this variation could also influence the efficacy of complex III inhibitors eliciting mitochondrially-mediated apoptosis.

A9664G causes the amino acid substitution E153G in MT-CO3. Complex IV, which is the terminal enzyme of the MRC that catalyses the electron transfer from cytochrome $\mathrm{c}$ to oxygen, which (like in complex III) is also coupled to proton translocation across the inner mitochondrial membrane. MT-CO3 forms a homodimer at the centre of complex IV. Although MT-CO3 does not house any active sites/binding pockets, through its interactions with MT-CO1 and various nuclear encoded subunits e.g. COX6A, it is thought to play a key role in the formation and therefore stabilisation of complex IV dimer [41]. In wild type MT-CO3, E153 occupies a position on the surface of MT-CO3 and interacts with alanine at position 13 of the nuclear-encoded subunit COX6A (Figure 6A). The E153G substitution is predicted to eliminate a hydrogen bond interaction (Figure 6B) that usually occurs between the large, negatively charged wild type glutamic acid residue of MT-CO3 and the smaller hydrophobic alanine of nuclear COX6A, potentially undermining the interaction between E153G and COX6A (Figure 6C, D). In summary, E153G could affect complex IV stability and therefore activity. Consistent with this view, knockdown of the subunit (although a more severe scenario than E153G) has been shown to reduce activity of complex IV [42-44].

T8821C causes an S to P substitution at position 99 of the MT-ATP6 polypeptide (Figure 7), which forms part of the F0 region of complex V. Complex V is the primary producer of ATP in eukaryotic cells, and in addition to the hydrophobic F0 region that traverses the inner mitochondrial membrane, it has a hydrophilic F1-ATPase region that protrudes like a 'lollipop' head into the mitochondrial matrix. In addition to MT-ATP6 (also known as the a-subunit) the F0 region is formed of an additional mtDNA-encoded subunit (MT-ATP8, also referred to as A6L-subunit), several nuclear encoded subunits (e, f, g, DAPIT, 2 hydrophobic alpha-helices of b), a proteolipid, and the c8-ring ('rotor'). The F0 and F1 regions are linked via the subunits OSCP, d, F6 and the hydrophilic portions of subunit $b$, referred to as the peripheral stalk. It is proposed that complex $\mathrm{V}$ drives the protons that arise in the inter membrane space (IMS) as a result of MRC activity through two half-channels formed by MT- ATP6 and the 'rotor'. In one half channel, conserved Glu58 in one of the c-subunits receives a proton from the IMS, which induces the c-ring to rotate, with the protonated Glu residues moving away from the static portions of the F0 region (the 'stator' which includes the two mtDNA-encoded subunits as well as components of the peripheral stalk). Once the protonated Glu residues have moved almost a full circle around the 'rotor', returning to the static portion of the F0 region, the other half channel provides an exit for the protons into the matrix. The resulting in deprotonation of the Glu residue, makes it ready to receive another round of protonation 
via the IMS. The mechanical energy created by the rotation, is transmitted via subunits gamma, sigma and epsilon (the 'shaft'), causing conformational changes in the alpha and beta subunits (the 'blades') of the F1 region, and

ultimately ATP hydrolysis (reviewed in [29]). Complex V is often referred to as a Brownian ratchet as rotation of the c-ring occurs through Brownian motion, but it is also referred to as a turbine as the enzyme has all the parts required to make a simple turbine (a rotor, stator, shaft and blades). Wild type MT-ATP6 is a 6-alpha helical bundle, whose tertiary folds are likely to be stabilised by the 12 proline residues (Figure S1). These proline-mediated kinks are likely to be indispensable for the positioning of MT-ATP6 close to the nuclear-encoded subunits of the c-ring rotor, as well as subunit b that forms part of the stator, and MT-ATP8, whose function of which is of yet unknown. The introduction of an additional proline residue, downstream of a conserved proline at position 94 of MT-ATP6 (Figure S1, Figure 7), is likely to affect thermodynamics of the whole subunit, and as a consequence, the numerous inter-helical interactions of MT-ATP6 mentioned above [45], this in turn could reduce complex $\mathrm{V}$ activity.

\subsection{Prevalence of functional candidates in patient bloods}

The detailed structural modelling suggests that altogether just 3 out of the 13 (23\%) BBM patients carried a single pathogenic variation: BBM4 carries T14819insTTCTATA in complex III, BBM12 carries E153G in complex IV, and BBM2 carries S99P in complex V. While the possibility that D-loop, rRNA, tRNA and synonymous variations could also affect the OXPHOS function through a variety of mechanisms, the current lack of in silico tools available to accurately predict the structural/functional effect of such variations on OXPHOS proteins limits informative interpretation.

\subsection{Summary and future prospects}

Although more patients are surviving primary breast cancer, more and more are developing secondary metastases, including to the brain. Although there are existing markers that relate to the likelihood of developing BBM (e.g. triple negative or Her2+, or ER-), more needs to be done to fully understand the process of BBM and develop additional markers that predict whether a patient will develop BBM. MtDNA variations are known to play a role in various diseases, including some cancers. With ever improving and more cost-effective sequencing technologies, an increasing number of mtDNA variations are being detected that are associated with disease. However, proving their role in processes like BBM remains difficult, one reason being that it is impossible to introduce single mtDNA variations into human mtDNA and observe direct phenotypic effects.

Our focus here was two-fold: to screen the bloods of BBM patients using an established and sensitive deep sequencing approach for mtDNA variation detection (and to predict their effect on mitochondrial function using an equally established 3D structural modelling approach [19]). We reveal that the vast majority of mtDNA protein-coding variations detected in the majority of patients tested are not predicted to have a major impact on mitochondrial function. This said, 3 of the mtDNA variations detected in three of the patients were predicted to have an impact at the level of OXPHOS protein structure. The potential multiple origins of the mtDNA variations (e.g. neutrophil, monocyte, myeloid dendritic, natural killer, $\mathrm{T}$ and B [49], as well tumour circulating cells [4]) detected in the bloods, as well as the different selective pressures which may act upon them, however, make it difficult to fully predict their significance on the process of BBM (even if they are predicted to evoke a change on the OXPHOS system), and requires further investigation using matched primary tumour-blood-secondary tumour samples to confirm their origin. Other future work could include determining how the $3 \mathrm{mtDNA}$ variations correlate with 1) the predicted alterations in mitochondrial function using in vitro studies and 2) with increased risk of developing breast brain metastasis using a much larger cohort of patient samples. This study also serves as a resource by highlighting a list of several mtDNA variations which are predicted to be non-functional and therefore not worthy of further investigation, which should help scientists and clinicians avoid wasting resources. Such a systematic approach of analyzing mtDNA variations could help improve 
our understanding of their role in the process of BBM as well as aid the development of markers that predict whether patients develop BBM in the future.

Acknowledgments: Running costs were provided by the Institute of Biomedical and Biomolecular Science (IBBS-fund) and REL's salary was supported by the charity Brain Tumour Research. We would also like to gratefully thank John E. McGeehan for his discussion during the design of the project and comments and guidance on the structural section of this work; Geoffrey J. Pilkington and Helen L. Fillmore for the use of the Brain Tumour Research Centre facilities; Sajid Rafiq, and Will Tapper for their discussions during the design of the project, and Nikki J. Graham for providing the blood DNA samples and associated details.

Declaration of Interest: The authors declare no conflict of interest. The founding sponsors had no role in the design of the study; in the collection, analyses, or interpretation of data; in the writing of the manuscript, and in the decision to publish the results.

\section{References}

1. Berman, A.T.; Thukral, A.D.; Hwang, W.T.; Solin, L.J; Vapiwala, N. Incidence and patterns of distant metastases for patients with early-stage breast cancer after breast conservation treatment. Clin. Breast Cancer. 2013, 13, 88-94.

2. Gil-Gil, M.J.; Martinez-Garcia, M.; Sierra, A.; Conesa, G.; Del Barco, S.; González-Jimenez, S.; Villà, S. Breast cancer brain metastases: A review of the literature and a current multidisciplinary management guideline. Clin Transl Oncol. 2014, 16, 436-46.

3. Engel, J.; Eckel, R.; Aydemir, Ü.; Aydemir, S.; Kerr, J.; Schlesinger-Raab, A; Dirschedl, P.; Hölzel, D. Determinants and prognoses of locoregional and distant progression in breast cancer. Int. J. Radiat. Oncol. Biol. Phys. 2003, 55, 1186-95.

4. Fidler, IJ. The Biology of Brain Metastasis: Challenges for Therapy. Cancer J. 2015, 21, $284-93$.

5. Khan, S.; Fagerholm, R.; Rafiq, S.; Tapper, W.; Aittomäki, K.; Liu, J.; Blomqvist, C.; Eccles, D.; Nevanlinna, H. Polymorphism at $19 q 13.41$ predicts breast cancer survival specifically after endocrine therapy. Clin. Cancer Res. 2015, 21, 4086-96.

6. Fagerholm, R.; Schmidt, M.K.; Khan, S.; Rafiq, S.; Tapper, W.; Aittomäki, K.; Groco, D.; Heikkinen, T.; Muranen, T.A.; Fasching, P.A.; et al. The SNP rs6500843 in 16p13.3 is associated with survival specifically among chemotherapy-treated breast cancer patients. Oncotarget. 2015, 6, 7390-407.

7. Rizzuto, R.; De Stefani, D.; Raffaello, A.; Mammucari, C. Mitochondria as sensors and regulators of calcium signalling. Nat. Rev. Mol. Cell Biol. 2012, 13, 566-78.

8. Sena, L.A.; Chandel, N.S. Physiological roles of mitochondrial reactive oxygen species. Mol. Cell. 2012, 48, $158-66$.

9. Jeong, S.Y.; Seol, D.W. The role of mitochondria in apoptosis. BMB Rep. 2008, 41, 11-22.

10. Wang, C.; Youle, R.J. The role of mitochondria in apoptosis*. Annu. Rev. Genet. 2009, 43, 95-118.

11. Schon, E.A.; DiMauro, S.; Hirano, M. Human mitochondrial DNA: roles of inherited and somatic mutations. Nat. Rev. Genet. 2012, 13, 878-90.

12. Rossignol, R.; Faustin, B.; Rocher, C.; Malgat, M.; Mazat, J.P.; Letellier, T. Mitochondrial threshold effects. Biochem. J. 2003, 370, 751-62.

13. Chen, E.I. Mitochondrial dysfunction and cancer metastasis. J. Bioenerg. Biomembr. 2012, 44, 619-22.

14. Ishikawa, K.; Takenaga, K.; Akimoto, M.; Koshikawa, N.; Yamaguchi, A.; Imanishi, H.; Nahada, K.; Honma, Y.; Hayashi, J. ROS-generating mitochondrial DNA mutations can regulate tumor cell metastasis. Science. 2008, 320, 661-4.

15. Wang, Y.; Liu, V.W.S.; Tsang, P.C.K.; Chiu, P.M.; Cheung, A.N.Y.; Khoo, U.S.; Nagley, P.; Ngan, H.Y.S. Microsatellite instability in mitochondrial genome of common female cancers. Int. J. Gynecol. Cancer. 2006, 16, 259-66.

16. Bai, R.K.; Leal, S.M.; Covarrubias, D.; Liu, A.; Wong, L.J.C. Mitochondrial genetic background modifies breast cancer risk. Cancer Res. 2007, 67, 4687-94.

17. Czarnecka, A.M.; Krawczyk, T.; Plak, K.; Klemba, A.; Zdrozny, M.; Arnold, R.S.; Kofler, B.; Golik, P.; Szybinskia, A.; Lubinski, J.; Mossakowska, M.; Bartnik, E.; Petros, J.A. Mitochondrial genotype and breast cancer predisposition. Oncol. Rep. 2010, 24, 1521-34.

18. Canter, J.A.; Kallianpur, A.R.; Parl, F.F.; Millikan, R.C. Mitochondrial DNA G10398A polymorphism and invasive breast cancer in African-American women. Cancer Res. 2005, 65, 8028-33. 
19. Lloyd, R.E.; Keatley, K.; Littlewood, D.T.J.; Meunier, B.; Holt, W.V.; An, Q.; Higgins, S.C.; Polyzoidis, S.; Stephenson, K.F.; Ashkan, K.; Fillmore, H.L.; Pilkington, G.J.; McGeehan, J.E. Identification and functional prediction of mitochondrial complex III and IV mutations associated with glioblastoma. Neuro-oncology. 2015, $17,942-52$.

20. Lloyd, R.E.; McGeehan, J.E. Structural Analysis of Mitochondrial Mutations Reveals a Role for Bigenomic Protein Interactions in Human Disease. PLoS One. 2013, 8, e69003.

21. Eccles, D.; Gerty, S.; Simmonds, P.; Hammond, V.; Ennis, S.; Altman, D.G. Prospective study of Outcomes in Sporadic versus Hereditary breast cancer (POSH): study protocol. BMC Cancer. 2007, 7, 160.

22. Rafiq, S.; Tapper, W.; Collins, A.; Khan, S.; Politopoulos, I.; Gerty, S.; Blomqvist, C.; Couch, F.J.; Nevanlinna, H.; Liu, J.; Eccles, D. Identification of inherited genetic variations influencing prognosis in early-onset breast cancer. Cancer Res. 2013, 73, 1883-91.

23. Andrews, R.M.; Kubacka, I.; Chinnery, P.F.; Lightowlers, R.N.; Turnbull, D.M.; Howell, N. Reanalysis and revision of the Cambridge reference sequence for human mitochondrial DNA. Nat. Genet. 1999, $23,147$.

24. Li, H.; Durbin, R. Fast and accurate short read alignment with Burrows-Wheeler transform. Bioinformatics. 2009, 25, 1754-60.

25. McKenna, A.; Hanna, M.; Banks, E.; Sivachenko, A.; Cibulskis, K.; Kernytsky, A.; Garimella, K.; Altshuler, D.; Gabriel, S.; Daly, M.; DePristo, M.A. The genome analysis toolkit: A MapReduce framework for analyzing next-generation DNA sequencing data. Genome Res. 2010, 20, 1297303.

26. DePristo, M.A.; Banks, E.; Poplin, R.; Garimella, K.V; Maguire, J.R.; Hartl, C.; Philippakis, A.A.; del Angel, G.; Rivas, M.A.; Hanna, M.; et al. A framework for variation discovery and genotyping using next-generation DNA sequencing data. Nat. Genet. 2011, 43, 491-8.

27. Ju, Y.S.; Alexandrov, L.B.; Gerstung, M.; Martincorena, I.; Nik-Zainal, S.; Ramakrishna, M.; Davies, H.R.; Papaemmanuil, E.; Gundem, G.; Shlien, A.; et al. Origins and functional consequences of somatic mitochondrial DNA mutations in human cancer. Elife. 2014, 3, e02935.

28. Zhu, J.; Vinothkumar, K.R.; Hirst, J. Structure of mammalian respiratory complex I. Nature. 2016, 536, 354-8.

29. Zhou, A.; Rohou, A.; Schep, D.G.; Bason, J.V.; Montgomery, M.G.; Walker, J.E.; Grigorieffniko, N.; Rubinstein, J.L. Structure and conformational states of the bovine mitochondrial ATP synthase by cryo-EM. Elife. 2015, 4, e10180.

30. Gao, X.; Wen, X.; Esser, L.; Quinn, B.; Yu, L.; Yu, C.A.; Xia, D. Structural Basis for the Quinone Reduction in the bc1 Complex: A Comparative Analysis of Crystal Structures of Mitochondrial Cytochrome bc1 with Bound Substrate and Inhibitors at the Qi Site. Biochemistry. 2003, 42, 9067-80.

31. Iwata, S.; Lee, J.W.; Okada, K.; Lee, J.K.; Iwata, M.; Rasmussen, B.; Link, T.A.; Ramaswamy, S.; Jap, B.K. Complete structure of the 11-subunit bovine mitochondrial cytochrome bc1 complex. Science. 1998, $281,64-71$.

32. Muramoto, K.; Hirata, K.; Shinzawa-Itoh, K.; Yoko-o, S.; Yamashita, E.; Aoyama, H.; Tsukihara, T.; Yoshikawa, S. A histidine residue acting as a controlling site for dioxygen reduction and proton pumping by cytochrome c oxidase. Proc. Natl. Acad. Sci. U.S.A. 2007, 104, 7881-6.

33. Larkin, M.; Blackshields, G.; Brown, N.; Chenna, R.; McGettigan, P.; McWilliam, H.; Valentin, F.; Wallace, I.M.; Wilm, A.; Lopez, R.; Thompson, J.D.; Gibson, T.J.; Higgins, D.G. ClustalW and ClustalX version 2. Bioinformatics. 2007, 23, 2947-8.

34. Gouet, P.; Courcelle, E.; Stuart, D.I.; Metoz, F. ESPript: analysis of multiple sequence alignments in PostScript. Bioinformatics. 1999, 15, 305-8.

35. Emsley, P.; Lohkamp, B.; Scott, W.G.; Cowtan, K. Features and development of Coot. Acta. Crystallogr. Sect. D Biol. Crystallogr. 2010, 66, 486-501.

36. Rostami, R.; Mittal, S.; Rostami, P.; Tavassoli, F.; Jabbari, B. Brain metastasis in breast cancer: a comprehensive literature review. J. Neurooncol. 2016, 127, 407-14.

37. Wiseman, H.; Halliwell, B. Damage to DNA by reactive oxygen and nitrogen species: role in inflammatory disease and progression to cancer. Biochem. J. 1996, 313, 17-29.

38. Akouchekian, M.; Houshmand, M.; Hemati, S.; Ansaripour, M.; Shafa, M. High rate of mutation in mitochondrial DNA displacement loop region in human colorectal cancer. Dis. Colon Rectum. 2009, 52, 526-30. 39. Fliss, M.S.; Usadel, H.; Caballero, O.L.; Wu, L.; Buta, M.R.; Eleff, S.M.; Jen, J.; Sidransky, D. Facile detection of mitochondrial DNA mutations in tumors and bodily fluids. Science. 2000, 287, 2017-9.

URL: http://mc.manuscriptcentral.com/gdna Email: desalle@amnh.org 
40. Parrella, P.; Xiao, Y.; Fliss, M.; Sanchez-Cespedes, M.; Mazzarelli, P.; Rinaldi, M.; Nicol, T.; Gabrielson, E.; Cuomo, D.; Cohen, D.; et al. Detection of mitochondrial DNA mutations in primary breast cancer and fine-needle aspirates. Cancer Res. 2001, 61, 7623-6.

41. Sedlák, E.; Robinson, N.C. Sequential dissociation of subunits from bovine heart cytochrome c oxidase by urea. Biochemistry. 2009, 48, 8143-50.

42. Quintens, R.; Singh, S.; Lemaire, K.; de Bock, K.; Granvik, M.; Schraenen, A.; Vroegrijk, I.O.C.M; Costa, V.; van Noten, P.; Lambrechts, D.; et al. Mice Deficient in the Respiratory Chain Gene Cox6a2 Are Protected against High-Fat Diet-Induced Obesity and Insulin Resistance. PLoS One. 2013, 8, e56719.

43. Tamiya, G.; Makino, S.; Hayashi, M.; Abe, A.; Numakura, C.; Ueki, M.; Tanaka, A.; Ito, C.; Tohimori, K.; Ogawa, N.; et al. A mutation of COX6A1 causes a recessive axonal or mixed form of Charcot-Marie-Tooth disease. Am. J. Hum. Genet. 2014, 95, 294-300.

44. Fornuskova, D.; Stiburek, L.; Wenchich, L.; Vinsova, K.; Hansikova, H.; Zeman, J. Novel insights into the assembly and function of human nuclear-encoded cytochrome c oxidase subunits 4, 5a, 6a, 7a and 7b. Biochem. J. 2010, 428, 363-74.

45. Schmidt, T.; Situ, A.J.; Ulmer, T.S. Structural and thermodynamic basis of proline-induced transmembrane complex stabilization. Sci Rep. 2016, 6, 29809.

46. Kenny TC, Hart P, Ragazzi M, Sersinghe M, Chipuk J, Sagar MA, Eliceiri KW, LaFramboise T, Grandhi S, Santos J, Riar AK, Papa L, D'Aurello M, Manfredi G, Bonini MG, Germain D. Selected mitochondrial DNA landscapes activate the SIRT3 axis of the UPR $(\mathrm{mt})$ to promote metastasis. Oncogene. 2017 Apr 3. doi: 10.1038/onc.2017.52. [Epub ahead of print] PubMed PMID: 28368421.

47. Milne I, Stephen G, Bayer M, Cock PJ, Pritchard L, Cardle L, Shaw PD, Marshall D. Using Tablet for visual exploration of second-generation sequencing data. Brief Bioinform. 2013 Mar;14(2):193-202.

48. Song Z, Laleve A, Vallières C, McGeehan JE, Lloyd RE, Meunier B. Human Mitochondrial Cytochrome b Variants Studied in Yeast: Not All Are Silent Polymorphisms. Hum Mutat. 2016 Sep;37(9):933-41.

49. Zhang P, Samuels DC, Wang J, Zhao S, Shyr Y, Guo Y. Mitochondria single nucleotide variation across six blood cell types. Mitochondrion. 2016 May;28:16-22.

Supplementary Materials: Figure S1. Location of non-synonymous amino acid substitution sites following the alignments of human (rCRS) against the bovine homologous mtDNA-encoded polypeptide sequences. Figure S2. Comparison of the wild type $m t-c y b$ and MT-CYB sequences against the variant sequences which contain a 6-nuclotide insertion at position 14819. Table S1: Details of the breast-to-brain metastasis patients from which whole-blood mitochondrial genomes were derived. Table S2. Long PCR Primer details. Table S3. Similarity of human sequences vs the homologous structural models used to analyse the effect mtDNA-encoded OXPHOS variations identified in breast-to-brain metastasis bloods. Table S4. Details of each mtDNA variation identified in breast-brain metastasis blood samples including location, average heteroplasmy and prevalence. Table S5. Reported disease associations of each mtDNA variation identified from breast-to-brain metastasis patient bloods. 
Tables with captions

Table 1. Summary of sequencing read statistics used to generate new complete breast-to-brain metastasis blood mtDNAs.

\begin{tabular}{cccccc}
\hline Sample ID & $\begin{array}{c}\text { Total number of } \\
\text { reads }\end{array}$ & $\begin{array}{c}\text { Number of reads } \\
\text { mapped }\end{array}$ & $\begin{array}{c}\text { Mean read } \\
\text { length }\end{array}$ & $\begin{array}{c}\text { Reads Mapped } \\
\text { (\%) }\end{array}$ & $\begin{array}{c}\text { Mean } \\
\text { DOC/nt }\end{array}$ \\
\hline BBM1 & 1286809 & 1236479 & 150 & 96 & 11255 \\
BBM2 & 1652912 & 1590565 & 150 & 96 & 14471 \\
BBM3 & 1398435 & 1341546 & 151 & 96 & 12236 \\
BBM4 & 1733369 & 1656272 & 150 & 96 & 15133 \\
BBM5 & 1606805 & 1486669 & 150 & 93 & 13587 \\
BBM6 & 2080624 & 2034506 & 150 & 98 & 18515 \\
BBM7 & 1616133 & 1576150 & 150 & 98 & 14358 \\
BBM8 & 1540641 & 1468502 & 151 & 95 & 13398 \\
BBM9 & 1709391 & 1632630 & 150 & 96 & 14911 \\
BBM10 & 1796414 & 1737453 & 150 & 97 & 15804 \\
BBM11 & 1517582 & 1461889 & 150 & 96 & 13331 \\
BBM12 & 1709260 & 1607356 & 150 & 94 & 14655 \\
BBM13 & 1500821 & 1449294 & 150 & 97 & 13252 \\
Average & 1626861 & 1559947 & 150.2 & 96 & 14223.6 \\
Standard & 196609 & 196351 & 0.3 & 1.4 & 1781.3 \\
Deviation & & & & & \\
\hline
\end{tabular}

Abbreviation: nt - nucleotide, DOC - depth of coverage

Table 2. Heteroplasmy, prevalence of non-synonymous variations in the mtDNA complex genes identified within the mtDNA landscapes of breast-to-brain blood samples.

\begin{tabular}{ccccccc}
\hline Locus & $\begin{array}{c}\text { Specific } \\
\text { Nucleotide } \\
\text { Variant }\end{array}$ & $\begin{array}{c}\text { Amino Acid } \\
\text { change }\end{array}$ & $\begin{array}{c}\text { Average } \\
\text { heteroplasmy } \mathbf{( \% \pm} \\
\text { SD) }\end{array}$ & $\begin{array}{c}\text { \% } \\
\text { Hmt } \\
\text { DB }\end{array}$ & $\begin{array}{c}\text { \% } \\
\text { BBM }\end{array}$ & $\begin{array}{c}\text { Specific } \\
\text { Sample } \\
\text { IDs }\end{array}$ \\
(BBM)
\end{tabular}


2

Grey shading indicates variation classed as functional at the level of protein structural changes. The unshaded variations are classified as non-functional. \%hmtDB and \%BBM indicate prevalence in the human mitochondrial database and BBM cohort (this study), respectively. *Signifies homoplasmic (pure) mutant (see materials and methods). 
Figures with captions

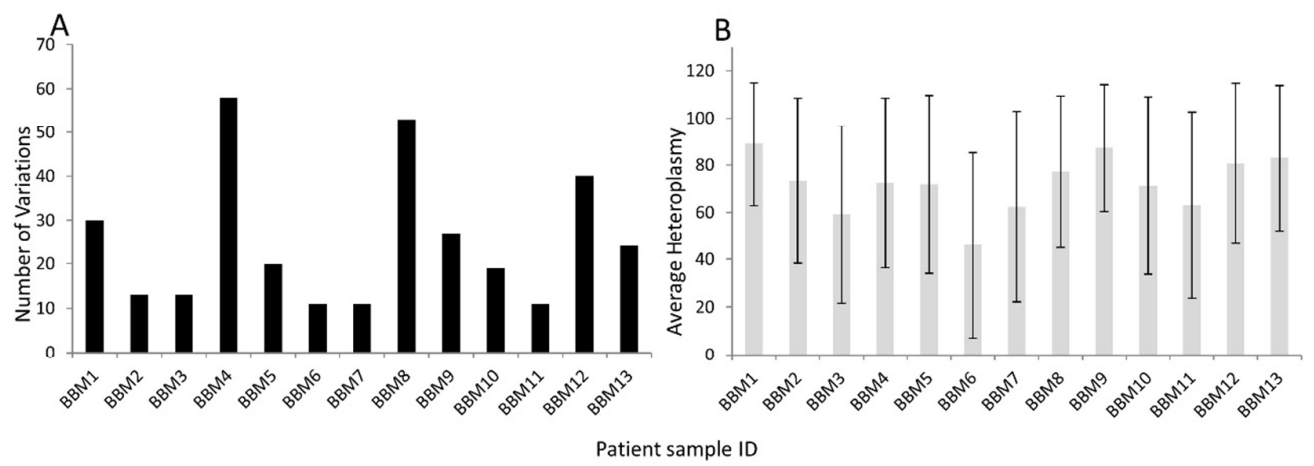

Figure 1. Total number (A) and average heteroplasmy (B) of mtDNA variations in breast-to-brain metastasis patient blood mtDNAs. Error bars indicate standard deviation.

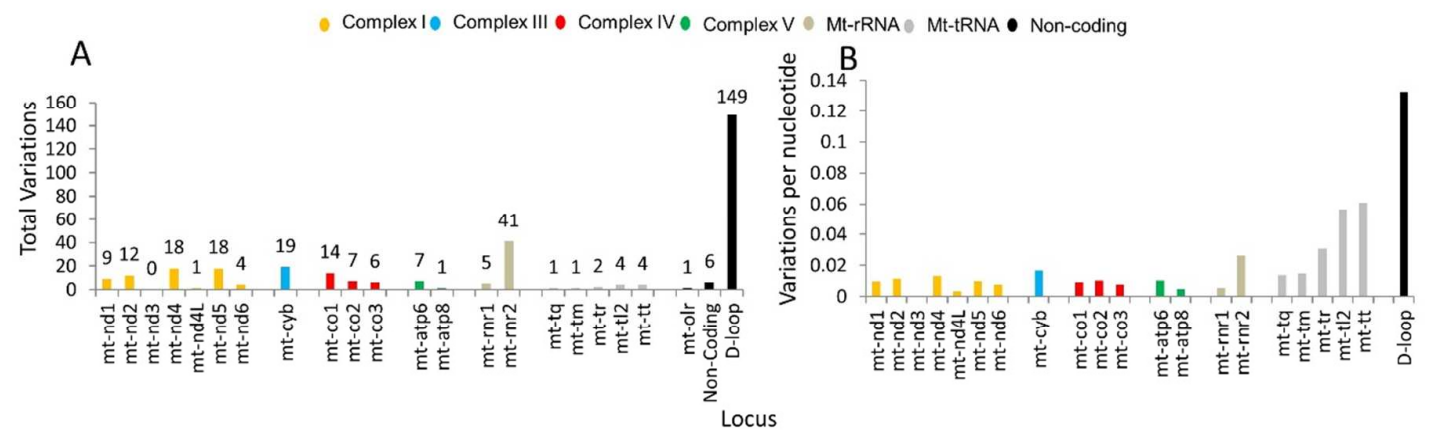

Figure 2. Presence of variations in breast-to-brain metastasis patient blood across mtDNA loci. (A) Total number of mtDNA variations identified in both coding and non-coding loci. (B) Total number of variations expressed per nucleotide in each of the mtDNA loci.

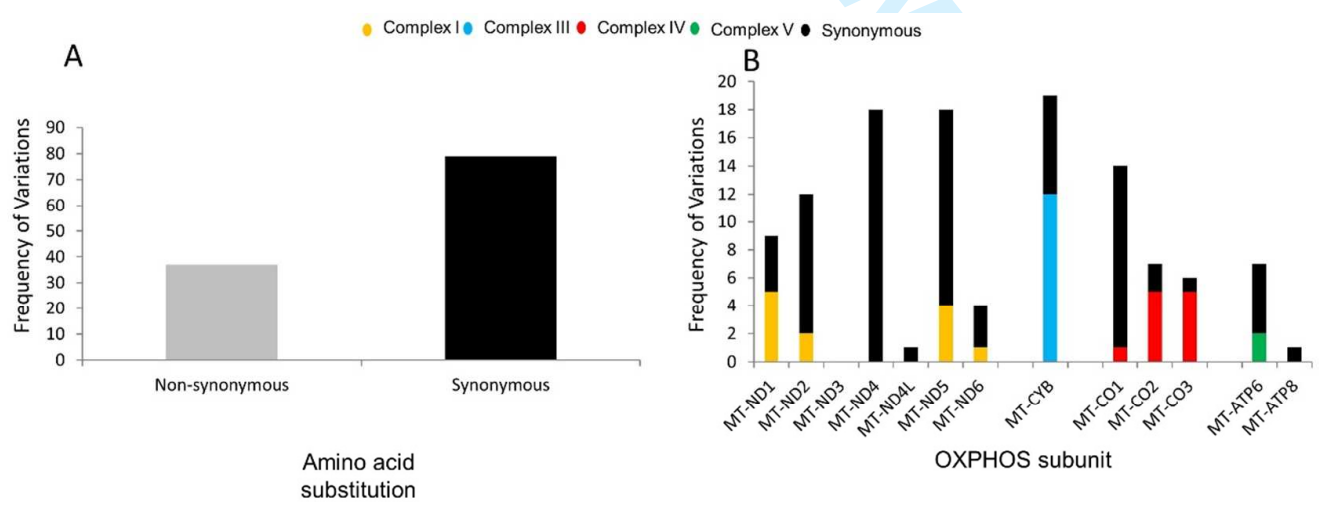

Figure 3. Pattern of synonymous and non-synonymous mtDNA variations in breast-to-brain blood mtDNAs. (A) Total and (B) across loci. 
map of non-synonymous variations identified in the mitochondrial OXPHOS proteins of breast-to-brain metastasis patient blood mtDNAs. Variations mapped to $\mathrm{T}$. thermophilus complex I homologues [PDB:4HEA], B. taurus complex III homologue [PDB:1BE3], B. taurus complex IV homologue [PDB:2EIJ] and B. taurus complex V homologoue [PDB: 5ARA]. The carbon alphas of residues that are conserved and non-conserved between the human sequences and the bovine models are highlighted as red and blue spheres respectively, including for complex I eventhough the T. thermophilus structure is shown. The conserved residue N150D is not shown as there is no equivalent residue present in $4 \mathrm{HEA}$

Complex I Complex III

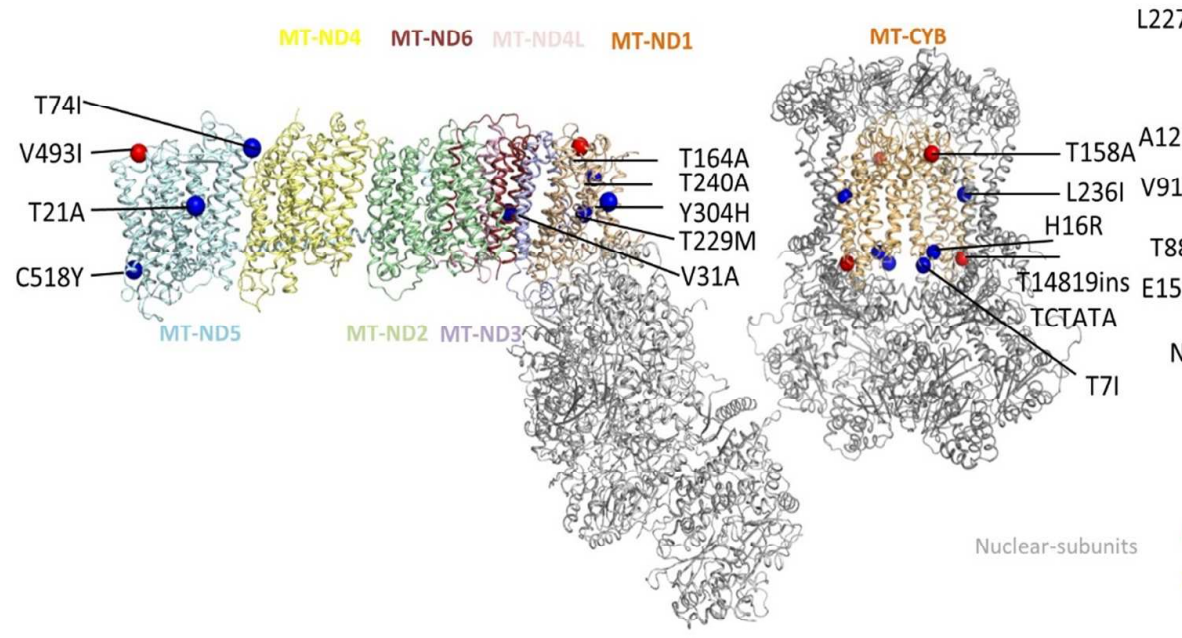

- Conserved mutation site

- Non-conserved mutation site 
A
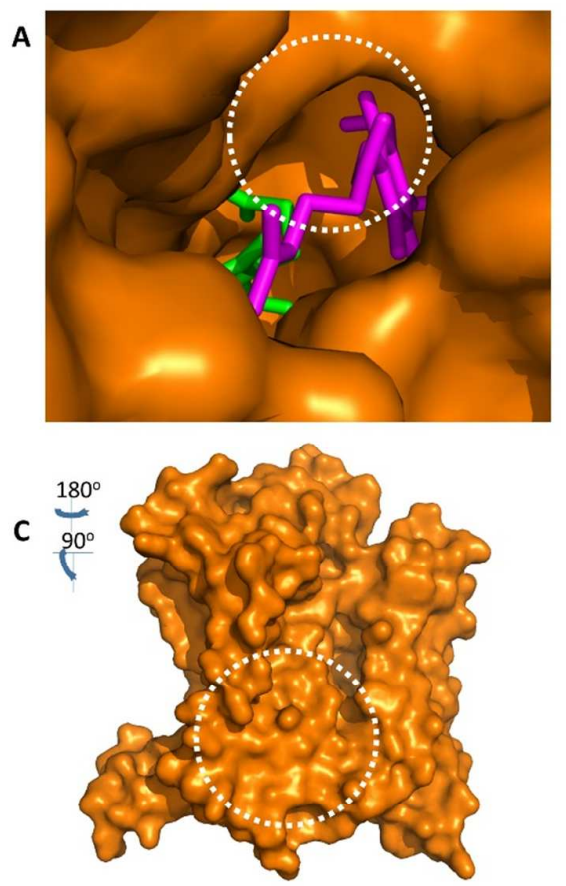

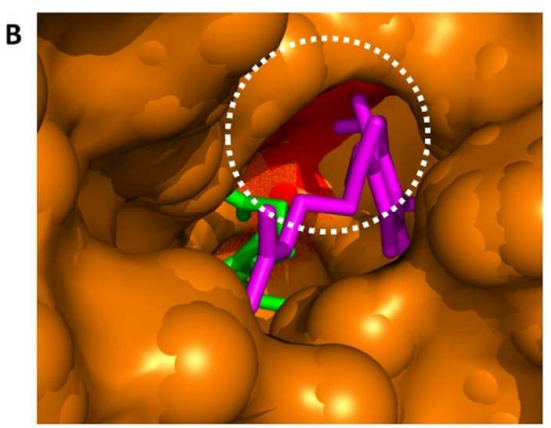

D

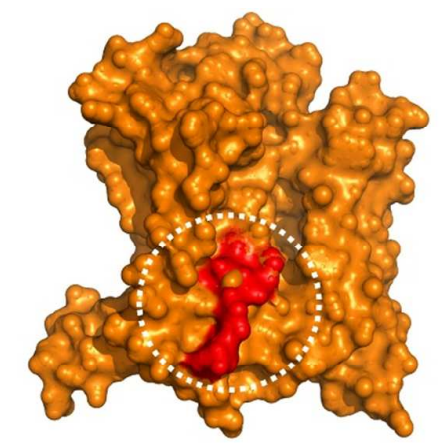

Figure 5. Structural consequences of the class 3 binding pocket variation 14819insTCTATA. The variation causes a serine to phenylalanine substitution and the addition of two amino acids at positions 25-27 of the complex III protein MT-CYB. MT-CYB is rendered as a space filling model (orange), with (A) the wild type Qi-site shown with the deeply buried heme (green) and bound ubiquinone (magenta; PDB 1NTZ). (B) The amino acid changes induced by the variation occur in the vicinity of the Qi-site (in red). (C) The surface of the wild type MT-CYB monomer. (D) The variant is likely to cause local mis-folding of MT-CYB (in red), disrupting its interaction with surrounding nuclear encoded subunits. Such changes to the MT-CYB Qi-site and surface are likely to interfere with ubiquinone/ubiquinol binding, and ultimately complex III activity.

A

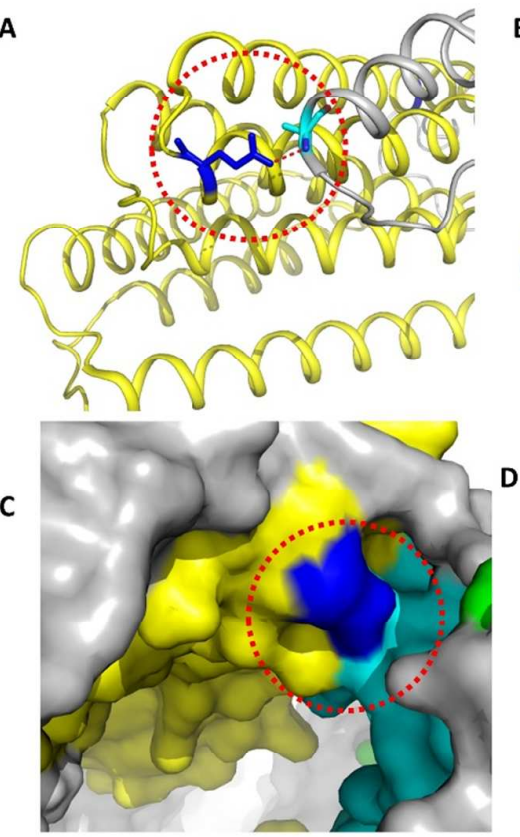

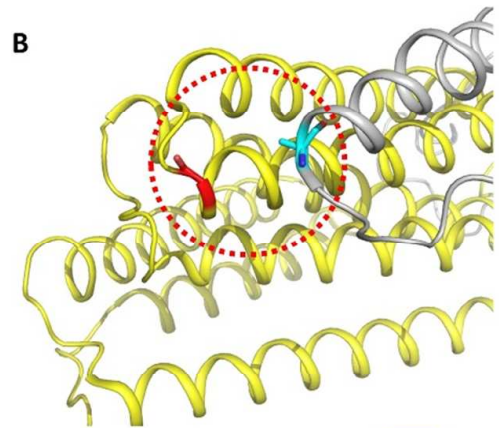

D

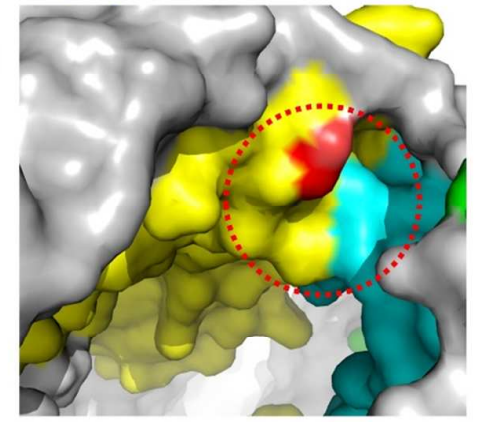


Figure 6. Structural consequences of the class 4 interaction variation A9664G. (A) Wild type E153 (blue sticks) in MT-CO3 (yellow ribbons) forms an interaction with A13 (cyan sticks) in COX6A (grey ribbon). (B) The variant G153 (red sticks) results in the loss of a hydrogen bond with A13. (C) The wild type MT-CO3 is shown as a surface model, showing E153 (blue) forms a tight interaction with A13 of COX6A (cyan). (D) Surface illustration of variant G153 (red), which no longer interacts with COX6A, which could affect the stability of complex IV.

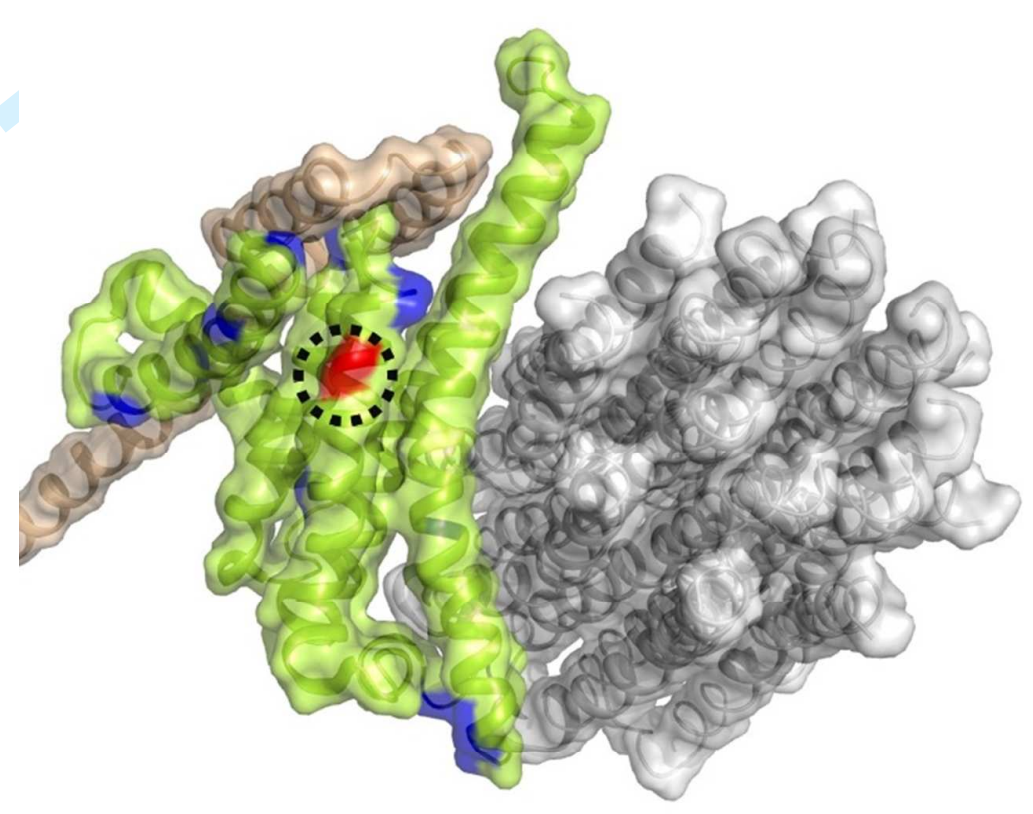

Figure 7. Structural consequences of the class 4 variation T8821C. Wild type MT-ATP6 (light green) is shown as a surface model and is formed of six kinked alpha-helices that stabilise the subunit and allow it to form intricate inter-helical interactions with subunit b (wheat), MT-ATP8 (not shown) and the c-ring (grey). Together with the hydrophobic portions of subunit b and MT-ATP8, MT-ATP6 form the static part of the F0 region, with the c-ring forming the rotary portion. It is likely that several proline residues (blue), conserved between the human sequence and the bovine model, are responsible for the kinks observed in MT-ATP6. The substitution of a serine for a proline (red) at position 99 in one of the helices, is likely to interfere with tertiary fold and destabilise the protein and disrupt its interactions, influencing complex $\mathrm{V}$ activity. 
Table 1. Summary of sequencing read statistics used to generate new complete breast-to-brain metastasis blood mtDNAs.

\begin{tabular}{cccccc}
\hline Sample ID & $\begin{array}{c}\text { Total number of } \\
\text { reads }\end{array}$ & $\begin{array}{c}\text { Number of reads } \\
\text { mapped }\end{array}$ & $\begin{array}{c}\text { Mean read } \\
\text { length }\end{array}$ & $\begin{array}{c}\text { Reads Mapped } \\
\text { (\%) }\end{array}$ & $\begin{array}{c}\text { Mean } \\
\text { DOC/nt }\end{array}$ \\
\hline BBM1 & 1286809 & 1236479 & 150 & 96 & 11255 \\
BBM2 & 1652912 & 1590565 & 150 & 96 & 14471 \\
BBM3 & 1398435 & 1341546 & 151 & 96 & 12236 \\
BBM4 & 1733369 & 1656272 & 150 & 96 & 15133 \\
BBM5 & 1606805 & 1486669 & 150 & 93 & 13587 \\
BBM6 & 2080624 & 2034506 & 150 & 98 & 18515 \\
BBM7 & 1616133 & 1576150 & 150 & 98 & 14358 \\
BBM8 & 1540641 & 1468502 & 151 & 95 & 13398 \\
BBM9 & 1709391 & 1632630 & 150 & 96 & 14911 \\
BBM10 & 1796414 & 1737453 & 150 & 97 & 15804 \\
BBM11 & 1517582 & 1461889 & 150 & 96 & 13331 \\
BBM12 & 1709260 & 1607356 & 150 & 94 & 14655 \\
BBM13 & 1500821 & 1449294 & 150 & 97 & 13252 \\
Average & 1626861 & 1559947 & 150.2 & 96 & 14223.6 \\
Standard & 196609 & 196351 & 0.3 & 1.4 & 1781.3 \\
Deviation & & & & & \\
\hline
\end{tabular}

Abbreviation: nt - nucleotide, DOC - depth of coverage 
Table 2. Heteroplasmy, prevalence of non-synonymous variations in the mtDNA complex genes identified within the mtDNA landscapes of breast-to-brain blood samples.

\begin{tabular}{|c|c|c|c|c|c|c|}
\hline Locus & $\begin{array}{c}\text { Specific } \\
\text { Nucleotide } \\
\text { Variant }\end{array}$ & $\begin{array}{l}\text { Amino Acid } \\
\text { change }\end{array}$ & $\begin{array}{c}\text { Average } \\
\text { heteroplasmy }(\% \pm \\
\text { SD) }\end{array}$ & $\begin{array}{c}\% \\
\text { Hmt } \\
\text { DB }\end{array}$ & $\begin{array}{c}\% \\
\text { BBM }\end{array}$ & $\begin{array}{c}\text { Specific Sample } \\
\text { IDs (BBM) }\end{array}$ \\
\hline MT-ND1 & $A 3796 G$ & T164A & $99.6^{*}$ & 0.6 & 7.7 & 6 \\
\hline MT-ND1 & C3992T & $\mathrm{T} 229 \mathrm{M}$ & $99.2^{*}$ & 0.8 & 7.7 & 10 \\
\hline MT-ND1 & A4024G & $\mathrm{T} 240 \mathrm{~A}$ & $99.2^{*}$ & 0.7 & 7.7 & 10 \\
\hline MT-ND1 & T4216C & $\mathrm{Y} 304 \mathrm{H}$ & $99.4 \pm 0.3^{*}$ & 10.5 & 15.4 & 1 and 12 \\
\hline MT-ND2 & $A 4917 G$ & N150D & $98.6 \pm 0.9^{*}$ & 5.2 & 15.4 & 1 and 12 \\
\hline MT-ND5 & $A 12397 G$ & $\mathrm{~T} 21 \mathrm{~A}$ & $99.6^{*}$ & 0.5 & 7.7 & 5 \\
\hline MT-ND5 & C12557T & T74I & $98.4^{*}$ & 0.3 & 7.7 & 4 \\
\hline MT-ND5 & G13813A & V493I & $99.6^{*}$ & 0.1 & 7.7 & 5 \\
\hline MT-ND5 & G13889A & C518Y & $99.6^{*}$ & 0.1 & 7.7 & 10 \\
\hline MT-ND6 & A14582G & V31A & $99.6^{*}$ & 0.6 & 7.7 & 10 \\
\hline MT-CYB & C14766T & T7I & $98.9 \pm 0.8^{*}$ & 74.9 & 46.2 & $1 / 4 / 8 / 9 / 12 / 13$ \\
\hline MT-CYB & $A 14793 G$ & H16R & $99.6 \pm 0.0^{*}$ & 2.3 & 15.4 & 9 and 13 \\
\hline MT-CYB & $\begin{array}{c}\text { T14819insTTCTA } \\
\text { TA }\end{array}$ & S25 & 2.4 & - & 7.7 & 12 \\
\hline MT-CYB & A15218G & T158A & $100 \pm 0.0^{*}$ & 2 & 7.7 & 9 \\
\hline MT-CYB & C15452A & L236I & $99.4 \pm 0.9^{*}$ & 9.8 & 15.4 & 1 and 12 \\
\hline MT-CO1 & G6267A & A122T & $98.8^{*}$ & 0.2 & 7.7 & 2 \\
\hline MT-CO2 & T8265C & L227P & 24.8 & 0 & 7.7 & 4 \\
\hline MT-CO3 & C9469T & T88I & $100^{*}$ & 0.1 & 7.7 & 13 \\
\hline MT-CO3 & G9477A & V91I & $99.8 \pm 0.3^{*}$ & 4.2 & 15.4 & 9 and 13 \\
\hline MT-CO3 & $A 9664 G$ & E153G & $98.8^{*}$ & 0.1 & 7.7 & 4 \\
\hline MT-CO3 & A9667G & N154S & $99.2^{*}$ & 0.7 & 7.7 & 9 \\
\hline MT-ATP6 & T8821C & S99P & 83.2 & 0 & 7.7 & 2 \\
\hline MT-ATP6 & G8989A & A155T & 97.2 & 0 & 7.7 & 12 \\
\hline
\end{tabular}

Grey shading indicates variation classed as functional at the level of protein structural changes. The unshaded variations are classified as non-functional. \% $\mathrm{hmtDB}$ and \%BBM indicate prevalence in the human mitochondrial database and BBM cohort (this study), respectively. *Signifies homoplasmic (pure) mutant (see materials and methods). 


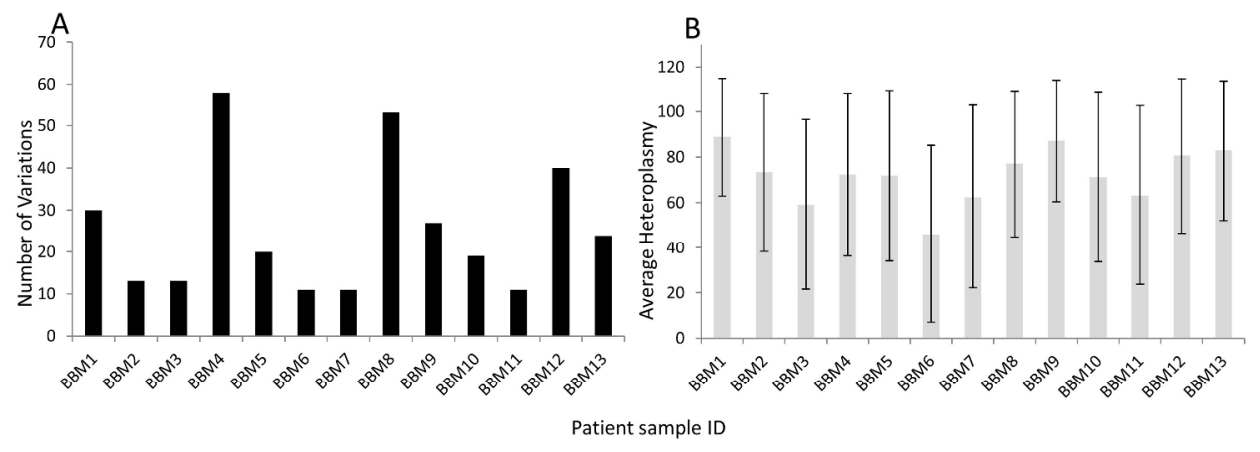
Total number (A) and average heteroplasmy (B) of mtDNA variations in breast-to-brain metastasis patient
blood mtDNAs. Error bars indicate standard deviation.

$254 \times 190 \mathrm{~mm}(300 \times 300$ DPI)

URL: http://mc.manuscriptcentral.com/gdna Email: desalle@amnh.org 


\begin{abstract}
. Presence of variations in breast-to-brain metastasis patient blood across mtDNA loci. (A) Total number of mtDNA variations identified in both coding and non-coding loci. (B) Total number of variations expressed per nucleotide in each of the mtDNA loci.
\end{abstract}

$254 \times 190 \mathrm{~mm}(300 \times 300 \mathrm{DPI})$

URL: http://mc.manuscriptcentral.com/gdna Email: desalle@amnh.org 


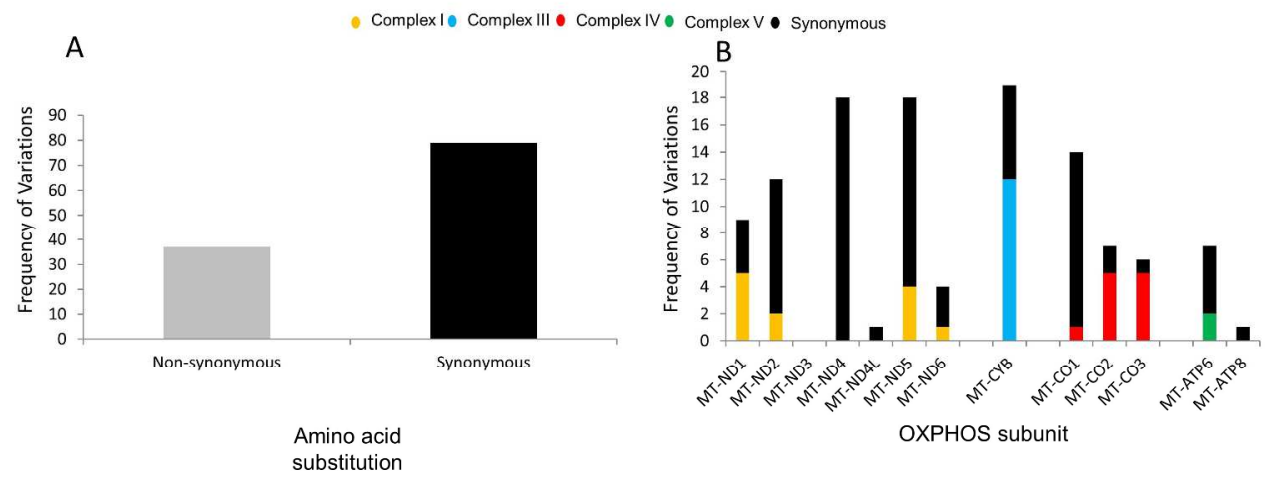

\section{Pattern of synonymous and non-synonymous mtDNA variations in breast-to-brain blood mtDNAs. (A) Total} and (B) across loci.

$254 \times 190 \mathrm{~mm}(300 \times 300 \mathrm{DPI})$ 
Global map of non-synonymous variations identified in the mitochondrial OXPHOS proteins of breast-to-brain metastasis patient blood mtDNAs. Variations mapped to $T$. thermophilus complex I homologues [PDB:4HEA], B. taurus complex III homologue [PDB:1BE3], B. taurus complex IV homologue [PDB:2EIJ] and $\mathrm{B}$. taurus complex $\mathrm{V}$ homologoue [PDB: 5ARA]. The carbon alphas of residues that are conserved and non-conserved between the human sequences and the bovine models are highlighted as red and blue spheres respectively, including for complex I eventhough the $T$. thermophilus structure is shown. The conserved residue N150D is not shown as there is no equivalent residue present in $4 \mathrm{HEA}$.

$254 \times 190 \mathrm{~mm}(300 \times 300 \mathrm{DPI})$ 

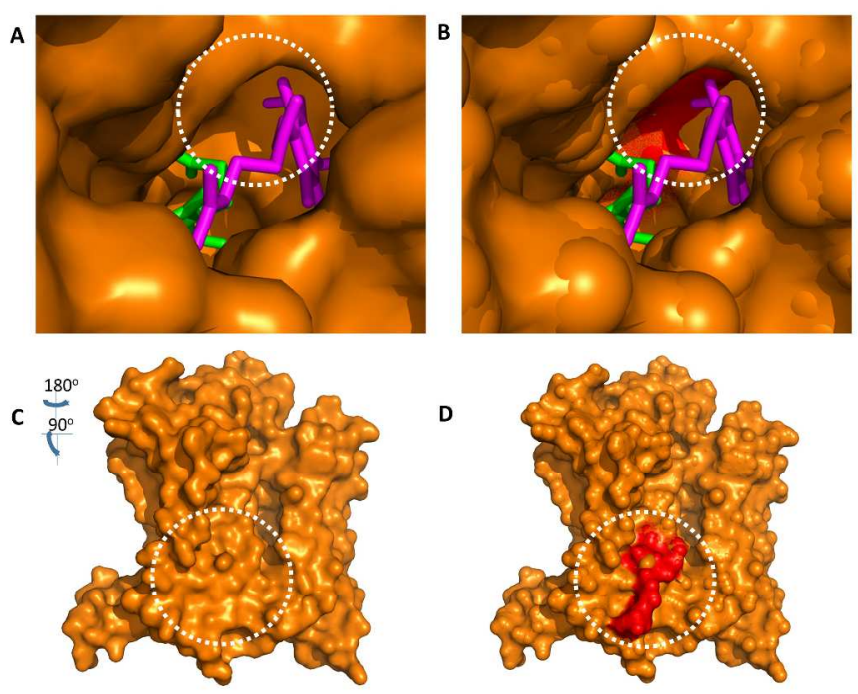

. Structural consequences of the class 3 binding pocket variation 14819insTCTATA. The variation causes a serine to phenylalanine substitution and the addition of two amino acids at positions 25-27 of the complex III protein MT-CYB. MT-CYB is rendered as a space filling model (orange), with (A) the wild type Qi-site shown with the deeply buried heme (green) and bound ubiquinone (magenta; PDB 1NTZ). (B) The amino acid changes induced by the variation occur in the vicinity of the Qi-site (in red). (C) The surface of the wild type MT-CYB monomer. (D) The variant is likely to cause local mis-folding of MT-CYB (in red), disrupting its interaction with surrounding nuclear encoded subunits. Such changes to the MT-CYB Qi-site and surface are likely to interfere with ubiquinone/ubiquinol binding, and ultimately complex III activity.

$338 \times 190 \mathrm{~mm}(300 \times 300 \mathrm{DPI})$ 
A
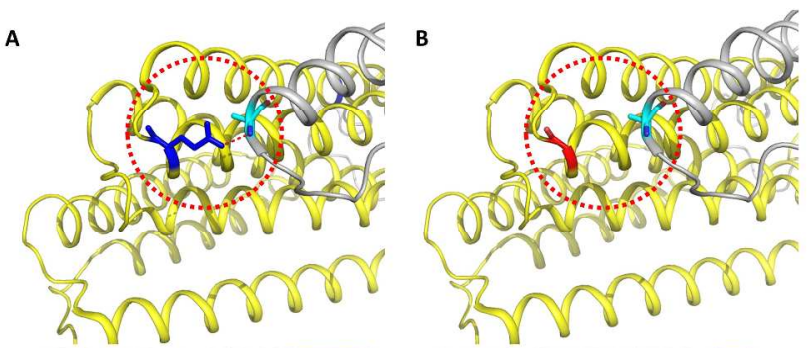

C
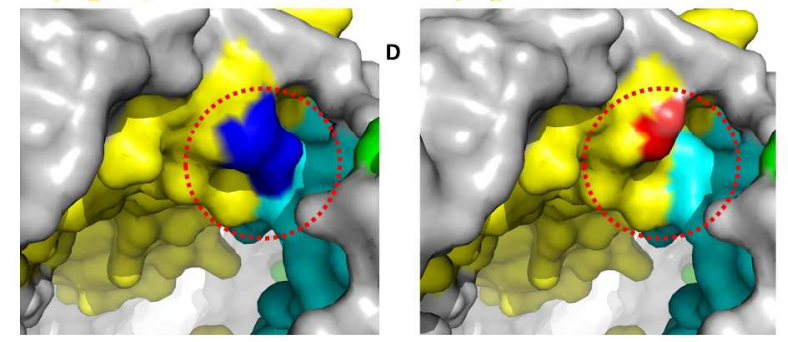

Structural consequences of the class 4 interaction variation A9664G. (A) Wild type E153 (blue sticks) in MTCO3 (yellow ribbons) forms an interaction with A13 (cyan sticks) in COX6A (grey ribbon). (B) The variant G153 (red sticks) results in the loss of a hydrogen bond with A13. (C) The wild type MT-CO3 is shown as a surface model, showing E153 (blue) forms a tight interaction with A13 of COX6A (cyan). (D) Surface illustration of variant G153 (red), which no longer interacts with COX6A, which could affect the stability of complex IV.

$338 \times 190 \mathrm{~mm}(300 \times 300 \mathrm{DPI})$ 
Structural consequences of the class 4 variation T8821C. Wild type MT-ATP6 (light green) is shown as a surface model and is formed of six kinked alpha-helices that stabilise the subunit and allow it to form intricate inter-helical interactions with subunit b (wheat), MT-ATP8 (not shown) and the c-ring (grey). Together with the hydrophobic portions of subunit $b$ and MT-ATP8, MT-ATP6 form the static part of the F0 region, with the c-ring forming the rotary portion. It is likely that several proline residues (blue), conserved between the human sequence and the bovine model, are responsible for the kinks observed in MT-ATP6. The substitution of a serine for a proline (red) at position 99 in one of the helices, is likely to interfere with tertiary fold and destabilise the protein and disrupt its interactions, influencing complex $\mathrm{V}$ activity.

$338 \times 190 \mathrm{~mm}(300 \times 300 \mathrm{DPI})$ 


\section{Supplementary Figures and Tables}

5

6

7

8

9

10

11

12

13

14

15

16

17

18

19

20

21

22

23

24

25

26

27

28

29

30

31

32

33

34

35

36

37

38

39

40

41

42

43

44

45

46

47

48

49

50

51

52

53

54

55

56

57

58

59

60

URL: http://mc.manuscriptcentral.com/gdna Email: desalle@amnh.org 
Table S1. Details of the breast-to-brain metastasis patients from which whole-blood mitochondrial genomes were derived.

\begin{tabular}{|c|c|c|c|c|c|c|c|c|}
\hline $\begin{array}{l}\text { Sample } \\
\text { ID }\end{array}$ & $\begin{array}{c}\text { POSSH } \\
\text { Sample No }\end{array}$ & NPI Score & ER & HER2 & PR & $\begin{array}{l}\text { BRCA } \\
\text { status }\end{array}$ & Chemotherapy* & $\begin{array}{c}\text { Adjuvant } \\
\text { Radiotherapy* }\end{array}$ \\
\hline BBM1 & 2003080226 & 5.7 & negative & negative & negative & BRCA1 & FEC, 6 cycles & No \\
\hline BBM2 & 2003120358 & 4 & positive & positive & positive & unknown & No & No \\
\hline BBM3 & 2004070684 & 3.6 & negative & not done & positive & unknown & No & No \\
\hline BBM4 & 2004080723 & $\mathrm{~N} / \mathrm{A}$ & negative & negative & negative & NMF & No & No \\
\hline BBM5 & 2005041135 & 5 & negative & not done & negative & unknown & FEC, 6 cycles & Yes \\
\hline BBM6 & 2005041147 & 5 & positive & negative & not done & unknown & EC + Docetaxel, 8 cycles & No \\
\hline BBM7 & 2008042970 & 7 & positive & positive & not done & unknown & FEC + Docetaxel, 6 cycles & No \\
\hline BBM8 & 2005051216 & 7 & positive & positive & negative & NMF & EC + Paclitaxel, 8 cycles & Yes \\
\hline BBM9 & 2006021768 & 6 & positive & negative & not done & unknown & FEC, 6 cycles & Yes \\
\hline BBM10 & 2007012323 & 5 & positive & negative & negative & unknown & E-CMF, 8 cycles & No \\
\hline BBM11 & 2007072618 & 5 & positive & negative & negative & unknown & E-CMF, 8 cycles & No \\
\hline BBM12 & 2008022929 & 7 & positive & positive & positive & unknown & EC + Docetaxel, 8 cycles & No \\
\hline BBM13 & 2008073036 & 5 & negative & positive & negative & unknown & $\begin{array}{c}\text { FEC, } 6 \text { cycles and } \\
\text { Docetaxel + } \\
\text { Trastuzumab, } 6 \text { cycles }\end{array}$ & No \\
\hline
\end{tabular}

Abbreviations: NPI - Nottingham Prognostic index; NMF-no mutation found; ER-oestrogen receptor status; HER2-HER2 status; PR-progesterone receptor status. FEC-fluorouracil, epirubicin and cyclophosphamide; E-CMF - epirubicin, cyclophosphamide, methotrexate and fluorouracil; EC - epirubicin and cyclophosphamide. *Prior to blood samples being taken. 
Table S2. Long PCR Primer details

\begin{tabular}{|c|c|c|c|c|}
\hline Name & Gene & $\begin{array}{l}\text { Nucleotide } \\
\text { position }\end{array}$ & Sequence $\left(5^{\prime}-3^{\prime}\right)$ & $\begin{array}{l}\text { Amplicon length } \\
\text { (bp) }\end{array}$ \\
\hline $\begin{array}{l}\text { CytbF } \\
\text { Human }\end{array}$ & $\begin{array}{l}\text { Mt-cyb_mt- } \\
\text { col }\end{array}$ & $15029-15049$ & $\begin{array}{l}\text { TTCCTACACATCGGGCGAGG } \\
\text { CCTCCTATGATGGCAAATACAG }\end{array}$ & 8573 \\
\hline LongR & & $7053-7078$ & CTCC & \\
\hline $\begin{array}{l}\text { HumanLong } \\
\text { F }\end{array}$ & $\begin{array}{l}\text { Mt-col_mt- } \\
\text { cyb }\end{array}$ & $6578-6603$ & $\begin{array}{l}\text { AGGAGACCCCATTCTATACCAA } \\
\text { CACC }\end{array}$ & 9128 \\
\hline CytbR & & $15731-15750$ & AGAATGAGGAGGTCTGCGGC & \\
\hline
\end{tabular}

The names, gene, nucleotide positions, sequences and expected amplicon lengths of PCR primers used to generate the overlapping long-amplicons. 
Table S3. Similarity of human sequences vs the homologous structural models used to analyse the effect mtDNA-encoded OXPHOS variations identified in breast-to-brain metastasis bloods. Complex III and IV similarities are reported elsewhere [1].

\begin{tabular}{|c|c|}
\hline Complex I & B. taurus [PDB ID:5LC5] \\
\hline Human Protein & Percentage Similarity \\
MT-ND1 & 78 \\
MT-ND2 & 63 \\
MT-ND3 & 73 \\
MT-ND4 & 74 \\
MT-ND4L & 73 \\
MT-ND5 & 70 \\
MT-ND6 & 61 \\
\hline
\end{tabular}

\begin{tabular}{c|c|}
\hline Complex V & B. taurus [PDB ID:5ARA] \\
\hline Human & Percentage Similarity \\
Protein & \\
MT-ATP6 & 77 \\
MT-ATP8 & Not available \\
\hline
\end{tabular}


Table S4. Details of the mtDNA variation landscapes identified in breast-brain metastasis blood samples including location, average heteroplasmy and prevalence.

\begin{tabular}{|c|c|c|c|c|c|c|}
\hline Locus & Specific Nucleotide Variant & $\begin{array}{l}\text { Amino Acid } \\
\text { change }\end{array}$ & $\begin{array}{c}\text { Average } \\
\text { heteroplasmy } \\
(\% \pm S D)\end{array}$ & $\begin{array}{c}\% \\
\text { Prevalence } \\
\text { in healthy } \\
\text { population } \\
\text { (Hmtdb) }\end{array}$ & $\begin{array}{l}\text { Prevalence in patient } \\
\text { samples (\%) }\end{array}$ & $\begin{array}{c}\text { Specific Sample IDs } \\
\text { (BBM) }\end{array}$ \\
\hline D-loop & A73G & - & $99.6 \pm 0.4$ & 76.9 & 46.2 & $1 / 4 / 8 / 9 / 12 / 13$ \\
\hline D-loop & A93G & - & $13.2 \pm 0.0$ & 2.5 & 7.7 & 6 \\
\hline D-loop & T146C & - & $99.8 \pm 0.3$ & 24.5 & 15.4 & $4 / 5$ \\
\hline D-loop & C150T & - & $99.6 \pm 0.6$ & 10.6 & 15.4 & $1 / 10$ \\
\hline D-loop & $T 152 C$ & - & $99.7 \pm 0.5$ & 28.6 & 23.1 & $4 / 8 / 9$ \\
\hline D-loop & A153G & - & 99.6 & 3.6 & 7.7 & 5 \\
\hline D-loop & T195C & - & 99.6 & 24.2 & 7.7 & 10 \\
\hline D-loop & A200G & - & 97.2 & 2.4 & 7.7 & 2 \\
\hline D-loop & $T 217 C$ & - & $99.4 \pm 0.3$ & 0.8 & 15.4 & $4 / 8$ \\
\hline D-loop & C299A & - & $14.5 \pm 1.8$ & 0 & 23.1 & $4 / 5 / 12$ \\
\hline D-loop & A301C & - & $15.4 \pm 3.9$ & 0 & 61.5 & $\begin{array}{c}1 / 2 / 3 / 5 / 7 / 10 / \\
11 / 12\end{array}$ \\
\hline D-loop & $A 302 A C$ & - & $13.1 \pm 3.0$ & - & 92.3 & $\begin{array}{c}1 / 2 / 3 / 4 / 5 / 6 / 7 / \\
9 / 10 / 11 / 12 / 13\end{array}$ \\
\hline
\end{tabular}




\begin{tabular}{|c|c|c|c|c|c|c|}
\hline D-loop & $A 302 A C C$ & - & 2.8 & - & 7.7 & 8 \\
\hline D-loop & С308T & - & $32 \pm 6.8$ & 0 & 15.4 & $8 / 12$ \\
\hline D-loop & СТзО9С & - & 2.0 & - & 7.7 & 12 \\
\hline D-loop & T310C & - & $75.7 \pm 4.0$ & 5.1 & 100 & All \\
\hline D-loop & T310TC & - & $18.2 \pm 4.5$ & - & 100 & All \\
\hline D-loop & T321C & - & 95.6 & 0.1 & 7.7 & 12 \\
\hline D-loop & С340T & - & 94.8 & 0.4 & 7.7 & 8 \\
\hline D-loop & C456T & - & 94.8 & 2.6 & 7.7 & 3 \\
\hline D-loop & $A 492 G$ & - & 14.4 & 0 & 7.7 & 5 \\
\hline D-loop & A508G & - & $87.6 \pm 8.5$ & 0.6 & 15.4 & $4 / 8$ \\
\hline D-loop & C510G & - & 12.8 & 0 & 7.7 & 8 \\
\hline D-loop & $A 512 C$ & - & 34.4 & 0.1 & 7.7 & 8 \\
\hline D-loop & G513C & - & $26.4 \pm 7.7$ & 0 & 15.4 & $3 / 10$ \\
\hline D-loop & GCA513G & - & $55.1 \pm 3.5$ & - & 15.4 & $3 / 10$ \\
\hline D-loop & G513A & - & 41.2 & 1.5 & 7.7 & 8 \\
\hline D-loop & G513GCA & - & 42.0 & - & 7.7 & 8 \\
\hline D-loop & G564C & - & 24.0 & 0 & 7.7 & 4 \\
\hline
\end{tabular}




\begin{tabular}{|c|c|c|c|c|c|c|}
\hline D-loop & C566A & - & 38.0 & 0 & 7.7 & 4 \\
\hline D-loop & $A 567 C$ & - & 42.3 & 0 & 7.7 & 4 \\
\hline D-loop & $A 567 A C$ & - & 42.8 & - & 7.7 & 4 \\
\hline D-loop & A16051G & - & $95.4 \pm 0.3$ & 2.6 & 15.4 & $4 / 8$ \\
\hline D-loop & $T 16092 C$ & & 93.2 & 1.3 & 7.7 & 4 \\
\hline D-loop & T16093C & -- & 92.4 & 5.6 & 7.7 & 8 \\
\hline D-loop & $C 16111 T$ & & 100 & 2.7 & 7.7 & 5 \\
\hline D-loop & $T 16126 C$ & - & $100 \pm 0$ & 11.9 & 15.4 & $1 / 12$ \\
\hline D-loop & G16129C & - & $98.4 \pm 0.6$ & 0.6 & 15.4 & $4 / 8$ \\
\hline D-loop & G16129A & -- & $99.8 \pm 0.3$ & 15.8 & 15.4 & $5 / 7$ \\
\hline D-loop & G16153A & - & 100 & 0.9 & 7.7 & 1 \\
\hline D-loop & CA16179C & - & $8.0 \pm 4.0$ & - & 15.4 & $4 / 8$ \\
\hline D-loop & $A 16181 A C$ & - & $8.4 \pm 2.9$ & - & 15.4 & $4 / 8$ \\
\hline D-loop & $A 16182 C$ & - & $70.8 \pm 3.4$ & 7.5 & 15.4 & $4 / 8$ \\
\hline D-loop & A16183C & - & $65.3 \pm 40.7$ & 13.6 & 23.1 & $4 / 6 / 8$ \\
\hline D-loop & $A 16183 A C$ & - & 12.0 & - & 7.7 & 6 \\
\hline D-loop & CT16188C & - & 8.8 & - & 7.7 & 6 \\
\hline
\end{tabular}




\begin{tabular}{|c|c|c|c|c|c|c|}
\hline D-loop & T16189C & - & $98 \pm 0.3$ & 29.6 & 15.4 & $4 / 8$ \\
\hline D-loop & C16192T & - & $98.8 \pm 0.6$ & 4.0 & 15.4 & $9 / 13$ \\
\hline D-loop & C16234T & - & 99.2 & 3.5 & 7.7 & 2 \\
\hline D-loop & C16256T & - & $98.1 \pm 2.8$ & 3.6 & 30.8 & $5 / 8 / 9 / 13$ \\
\hline D-loop & A16258C & - & 98.4 & 0.3 & 7.7 & 8 \\
\hline D-loop & C16270T & - & $92.8 \pm 8.5$ & 5.3 & 15.4 & $9 / 13$ \\
\hline D-loop & C16291T & & 98.8 & 2.8 & 7.7 & 1 \\
\hline D-loop & C16294T & - & $98.4 \pm 2.3$ & 9.3 & 15.4 & $1 / 12$ \\
\hline D-loop & C16296T & - & $98.8 \pm 0.6$ & 2.4 & 15.4 & $1 / 12$ \\
\hline D-loop & T16304C & - & $99.2 \pm 0.6$ & 6.2 & 15.4 & $3 / 12$ \\
\hline D-loop & T16311C & - & $99.3 \pm 0.6$ & 23.6 & 23.1 & $2 / 11 / 12$ \\
\hline D-loop & A16316G & -- & 99.6 & 1.0 & 7.7 & 7 \\
\hline D-loop & C16320T & - & 99.6 & 2.4 & 7.7 & 5 \\
\hline D-loop & $\mathrm{T} 16362 \mathrm{C}$ & - & $99.3 \pm 0.6$ & 16.0 & 23.1 & $3 / 4 / 8$ \\
\hline D-loop & A16399G & - & 100 & 2.6 & 7.7 & 9 \\
\hline D-loop & T16519C & - & $98.9 \pm 1.0$ & 66.9 & 53.8 & $1 / 4 / 5 / 6 / 7 / 10 / 12$ \\
\hline D-loop & G16526A & & 98.4 & 1.1 & 7.7 & 13 \\
\hline
\end{tabular}


Mitochondrial DNA

\begin{tabular}{|c|c|c|c|c|c|c|}
\hline RNR1 & G709A & - & $99.1 \pm 0.5$ & 12.8 & 23.1 & $1 / 9 / 12$ \\
\hline RNR1 & G930A & - & 99.2 & 2.2 & 7.7 & 12 \\
\hline RNR1 & G988A & - & 99.6 & 0.1 & 7.7 & 8 \\
\hline RNR2 & $A 1811 G$ & - & $99.8 \pm 0.3$ & 8.2 & 15.4 & $4 / 8$ \\
\hline RNR2 & G1888A & - & $99.4 \pm 0.3$ & 5.8 & 15.4 & $1 / 12$ \\
\hline RNR2 & $A 2581 G$ & - & 100 & 0.3 & 7.7 & 5 \\
\hline RNR2 & $A 2706 G$ & - & $98.7 \pm 0.6$ & 77.0 & 61.5 & $\begin{array}{c}1 / 2 / 4 / 8 / 9 / 11 / 12 / \\
13\end{array}$ \\
\hline RNR2 & G3010A & - & 99.2 & 16.2 & 7.7 & 6 \\
\hline RNR2 & AC3105A & & $56.3 \pm 0.5$ & - & 100 & All \\
\hline RNR2 & A3105T & - & $17.7 \pm 5.4$ & 0 & 69.2 & $\begin{array}{c}3 / 4 / 5 / 7 / 9 / 10 / \\
11 / 12 / 13\end{array}$ \\
\hline RNR2 & T3197C & - & $100 \pm 0.0$ & 4.2 & 15.4 & 9/ 13 \\
\hline RNR2 & G3226T & - & 39.2 & 0 & 7.7 & 8 \\
\hline RNR2 & T3229A & - & 42.8 & 0 & 7.7 & 8 \\
\hline RNR2 & T3229TA & - & 54.8 & - & 7.7 & 8 \\
\hline MT-ND1 & A3720G & Q138Q & $99.6 \pm 0$ & 0.6 & 15.4 & $4 / 8$ \\
\hline MT-ND1 & A3796G & T164A & 99.6 & 0.6 & 7.7 & 6 \\
\hline
\end{tabular}




\begin{tabular}{|c|c|c|c|c|c|c|}
\hline MT-ND1 & G3849A & L181L & 99.6 & 0.4 & 7.7 & 4 \\
\hline MT-ND1 & С3992T & T229M & 99.2 & 0.8 & 7.7 & 10 \\
\hline MT-ND1 & A4024G & $\mathrm{T} 240 \mathrm{~A}$ & 99.2 & 0.7 & 7.7 & 10 \\
\hline MT-ND1 & A4191C & P295P & 99.6 & 0 & 7.7 & 8 \\
\hline MT-ND1 & T4216C & Y304H & $99.4 \pm 0.3$ & 10.5 & 15.4 & $1 / 12$ \\
\hline MT-ND2 & T4553C & F28F & 99.6 & 0.2 & 7.7 & 4 \\
\hline MT-ND2 & T4736C & T89T & 99.2 & 0.1 & 7.7 & 4 \\
\hline MT-ND2 & $T 4838 \mathrm{C}$ & P123P & 98.8 & 0 & 7.7 & 7 \\
\hline MT-ND2 & A4917G & N150D & $98.6 \pm 0.9$ & 5.2 & 15.4 & $1 / 12$ \\
\hline MT-ND2 & T5004C & L179L & 100 & 0.9 & 7.7 & 10 \\
\hline MT-ND2 & G5147A & $\mathrm{T} 226 \mathrm{~T}$ & $99.8 \pm 0.3$ & 3.9 & 15.4 & $5 / 12$ \\
\hline MT-ND2 & A5390G & M307M & $100 \pm 0.0$ & 0.7 & 15.4 & $4 / 8$ \\
\hline MT-ND2 & T5426C & $\mathrm{H} 319 \mathrm{H}$ & $99.6 \pm 0.6$ & 0.9 & 15.4 & $4 / 8$ \\
\hline MT-ND4 & A10876G & L39L & $99.8 \pm 0.3$ & 1.1 & 15.4 & $4 / 8$ \\
\hline MT-ND4 & $A 11251 G$ & L164L & $99.4 \pm 0.9$ & 9.8 & 15.4 & $1 / 12$ \\
\hline MT-ND4 & T11353C & A198A & 99.6 & 0.2 & 7.7 & 9 \\
\hline MT-ND4 & A11467G & L236L & $98.7 \pm 1.2$ & 13.5 & 30.8 & $4 / 8 / 9 / 13$ \\
\hline
\end{tabular}




\section{Mitochondrial DNA}

\begin{tabular}{|c|c|c|c|c|c|c|}
\hline MT-ND4 & G11719A & G320G & $99.1 \pm 0.8$ & 75.3 & 53.8 & $1 / 4 / 7 / 8 / 9 / 12 / 13$ \\
\hline MT-ND4 & $A 11812 G$ & L351L & $99.2 \pm 0.0$ & 3.6 & 15.4 & $1 / 12$ \\
\hline $\begin{array}{l}\text { MT- } \\
\text { ND4L }\end{array}$ & C10619T & N50N & 99.6 & 0.1 & 7.7 & 13 \\
\hline MT-ND5 & G12372A & L12L & $99.4 \pm 1$ & 14.3 & 30.8 & $4 / 8 / 9 / 13$ \\
\hline MT-ND5 & A12397G & T21A & 99.6 & 0.5 & 7.7 & 5 \\
\hline MT-ND5 & C12557T & $\mathrm{T74I}$ & 98.4 & 0.3 & 7.7 & 4 \\
\hline MT-ND5 & T13020C & G228G & $99.2 \pm 1.1$ & 1.1 & 15.4 & $4 / 8$ \\
\hline MT-ND5 & G13368A & G344G & $99.4 \pm 0.3$ & 5.3 & 15.4 & $1 / 12$ \\
\hline MT-ND5 & A13419T & G361G & 22.0 & 0 & 7.7 & 13 \\
\hline MT-ND5 & T13617C & 14271 & $99.8 \pm 0.3$ & 4.1 & 15.4 & $9 / 13$ \\
\hline MT-ND5 & $T 13734 C$ & $\mathrm{~F} 466 \mathrm{~F}$ & $99.0 \pm 0.3$ & 0.6 & 15.4 & $4 / 8$ \\
\hline MT-ND5 & G13813A & V493I & 99.6 & 0.1 & 7.7 & 5 \\
\hline MT-ND5 & G13889A & C518Y & 99.6 & 0.1 & 7.7 & 10 \\
\hline MT-ND5 & A14145G & T603T & 98.8 & 0 & 7.7 & 11 \\
\hline MT-ND6 & A14233G & D147D & $99.2 \pm 0.6$ & 3.9 & 15.4 & $1 / 12$ \\
\hline MT-ND6 & C14365T & V103V & 99.2 & 0.6 & 7.7 & 10 \\
\hline MT-ND6 & A14582G & V31A & 99.6 & 0.6 & 7.7 & 10 \\
\hline
\end{tabular}




\begin{tabular}{|c|c|c|c|c|c|c|}
\hline MT-CYB & C14766T & T7I & $98.9 \pm 0.8$ & 74.9 & 46.2 & $1 / 4 / 8 / 9 / 12 / 13$ \\
\hline MT-CYB & A14793G & H16R & $99.6 \pm 0.0$ & 2.3 & 15.4 & $9 / 13$ \\
\hline MT-CYB & T14819TTCTATA & S25 & 2.4 & - & 7.7 & 12 \\
\hline MT-CYB & G14905A & M53M & $98.4 \pm 0.6$ & 5.5 & 15.4 & $1 / 12$ \\
\hline MT-CYB & A15218G & T158A & $100 \pm 0.0$ & 2.0 & 7.7 & 9 \\
\hline MT-CYB & C15452A & L236। & $99.4 \pm 0.9$ & 9.8 & 15.4 & $1 / 12$ \\
\hline MT-CYB & T15461C & L239L & $100 \pm 0.0$ & 0 & 7.7 & 12 \\
\hline MT-CYB & A15607G & K287K & $99.0 \pm 0.9$ & 5.5 & 15.4 & $1 / 12$ \\
\hline MT-CYB & C15661T & P305P & $99.6 \pm 0.0$ & 0.1 & 7.7 & 8 \\
\hline MT-CYB & C15833T & L363L & $99.2 \pm 0.0$ & 0.7 & 7.7 & 3 \\
\hline MT-CO1 & C6045T & L48L & $99.8 \pm 0.3$ & 0.6 & 15.4 & $4 / 8$ \\
\hline MT-CO1 & T6152C & V83V & $99.4 \pm 0.3$ & 0.7 & 15.4 & $4 / 8$ \\
\hline MT-CO1 & G6267A & $\mathrm{A} 122 \mathrm{~T}$ & 98.8 & 0.2 & 7.7 & 2 \\
\hline MT-CO1 & T6776C & $\mathrm{H} 291 \mathrm{H}$ & 99.2 & 2.2 & 7.7 & 5 \\
\hline MT-CO1 & C7028T & A375A & $99.4 \pm 0.6$ & 78.6 & 61.5 & $\begin{array}{c}1 / 2 / 4 / 8 / 9 / 11 / \\
12 / 13\end{array}$ \\
\hline MT-CO2 & T8265C & L227P & 24.8 & 0 & 7.7 & 4 \\
\hline MT-CO2 & A8266C & L227L & 32.0 & 0 & 7.7 & 4 \\
\hline
\end{tabular}




\begin{tabular}{|c|c|c|c|c|c|c|}
\hline MT-CO2 & T8267C & STOP>Q & 27.6 & 0 & 7.7 & 4 \\
\hline MT-CO2 & TAGC8267T & STOP & 3.7 & - & 7.7 & 4 \\
\hline MT-CO2 & A8268T & $S T O P>L$ & 26.1 & 0 & 7.7 & 4 \\
\hline MT-CO2 & G8269C & $S T O P>Y$ & 39.4 & 1.4 & 7.7 & 4 \\
\hline MT-CO2 & G8269A & STOP $>S T O P$ & 99.6 & 0 & 7.7 & 10 \\
\hline MT-CO3 & C9469T & T88I & 100 & 0.1 & 7.7 & 13 \\
\hline MT-CO3 & G9477A & V911 & $99.8 \pm 0.3$ & 4.2 & 15.4 & $9 / 13$ \\
\hline MT-CO3 & A9664G & E153G & 98.8 & 0.1 & 7.7 & 4 \\
\hline MT-CO3 & A9667G & N154S & 99.2 & 0.7 & 7.7 & 9 \\
\hline MT-CO3 & T9845C & $\mathrm{T} 213 \mathrm{~T}$ & 99.6 & 0.1 & 7.7 & 8 \\
\hline MT-ATP6 & $C 8622 A$ & P32P & 99.6 & 0 & 7.7 & 8 \\
\hline MT-ATP6 & G8697A & M57M & $99.8 \pm 0.3$ & 4.9 & 15.4 & $1 / 12$ \\
\hline MT-ATP6 & T8821C & S99P & 83.2 & 0 & 7.7 & 2 \\
\hline MT-ATP6 & G8989A & A155T & 97.2 & 0 & 7.7 & 12 \\
\hline MT-ATP6 & G8994A & L156L & 100 & 2.2 & 7.7 & 11 \\
\hline MT-ATP6 & G9123A & L199L & 100 & 6.1 & 7.7 & 10 \\
\hline MT-ATP8 & T8473C & P36P & 99.2 & 1.1 & 7.7 & 4 \\
\hline
\end{tabular}




\begin{tabular}{ccccccc}
\hline MT-OLR & G5746A & - & 96.8 & 0.1 & 7.7 & 2 \\
\hline NC & T5892A & - & 31.6 & 0 & 7.7 & 8 \\
NC & A5894C & - & 42.4 & 0.1 & 7.7 & 8 \\
NC & A5894ACC & - & 50.0 & - & 7.7 & 8 \\
NC & C8270T & - & 34.1 & 0.4 & 7.7 & 4 \\
NC & C8270CACCCCCTCT & - & 28.9 & - & 4.7 & 4 \\
NC & T8277TCTACCCCCC & - & 2.4 & - & 7.7 & 3 \\
\hline MT-TQ & T4336C & - & 99.6 & 1.1 & 7.7 & 4 \\
\hline MT-TM & A4435G & - & 100 & 0.1 & 7.7 & $1 / 12$ \\
\hline MT-TR & T10463C & - & $99.4 \pm 0.3$ & 5.0 & 15.4 & $4 / 8 / 9 / 13$ \\
\hline MT-TL2 & A12308G & - & $99.0 \pm 0.5$ & 13.5 & 30.8 & $4 / 8$ \\
\hline MT-TT & A15907G & - & $99.8 \pm 0.3$ & 0.6 & 15.4 & $1 / 12$ \\
MT-TT & G15928A & - & $99.6 \pm 0.6$ & 5.1 & 15.4 & 4
\end{tabular}

Abbreviations: ND - NADH dehydrogenase subunit, CYB - Cytochrome Bc1 complex subunit, CO - Cytochrome C oxidase subunit, ATP - ATP synthase subunit, OLR - Origin of light strand replication, RNR - Ribosomal RNA gene, HmtDB - Human mitochondrial database 
Table S5. Reported disease associations of each mtDNA variation identified from breast-to-brain metastasis patient bloods, as listed in MITOMAP (Green) and the human mitochondrial database (black)(date accessed $25^{\text {th }}$ of October 2016).

\begin{tabular}{|c|c|c|}
\hline $\begin{array}{l}\text { Locus/ } \\
\text { Human } \\
\text { Subunit } \\
\text { (Gene) }\end{array}$ & $\begin{array}{l}\text { Human } \\
\text { Nucleotide } \\
\text { Change } \\
\text { (Amino Acid } \\
\text { Change) }\end{array}$ & Disease Association \\
\hline \multirow[t]{16}{*}{ D-loop } & A73G & Thyroid tumours/ Prostate tumours \\
\hline & A93G & Thyroid cancer/ Breast cancer \\
\hline & T146C & $\begin{array}{l}\text { Elderly fibroblasts/ Elderly \& AD brains/ Prostate tumour/Ovarian } \\
\text { carcinoma/ POLG/PEO }\end{array}$ \\
\hline & C150T & $\begin{array}{c}\text { Elderly fibroblasts \& leukocytes/Lung tumour/Thyroid tumour/ Prostate } \\
\text { tumours/Leukocytes/ Lung cancer/ Thyroid cancer/ Prostate cancer/ } \\
\text { Oesophageal cancer/ MELAS/Obesity/Leber Hereditary Optic } \\
\text { Neuropathy/Oncocytic pituitary adenoma/Gastric carcinoma/Colorectal } \\
\text { cancer/Cardiomyopathy/Alzheimer/Noonan } \\
\text { syndrome/Epilepsy/Neuropathy/ Ataxia and retinitis } \\
\text { pigmentosa/Schizophrenia/Diabetes/Mitochondrial } \\
\text { disease/Glaucoma/GBM/Oral squamous cell carcinoma/Serous Ovarian } \\
\text { Cancer }\end{array}$ \\
\hline & $T 152 C$ & Elderly brains/ Elderly fibroblasts/ Ovarian carcinoma/ Breast tumour \\
\hline & A153G & $\begin{array}{c}\text { Thyroid tumor/Parkinson`s Disease/ Alzheimer's } \\
\text { Disease/Diabetes/Obesity/Oncocytic pituitary adenoma/Endometrial } \\
\text { carcinoma/Prostate cancer/Schizophrenia and bipolar disorder/Noonan } \\
\text { syndrome/Cardiomyopathy/Leber hereditary optic neuropathy/Breast } \\
\text { cancer/Esophageal cancer/Epilepsy/GBM }\end{array}$ \\
\hline & T195C & $\begin{array}{c}\text { Bipolar disorder/ Elderly/AD brains/ Lung tumour/ Thyroid tumour/ } \\
\text { Ovarian tumour/ Prostate tumour/ Glioblastoma/ Melanoma }\end{array}$ \\
\hline & A200G & $\begin{array}{c}\text { Renal oncocytoma/Gastric cancer/Colorectal } \\
\text { cancer/Cardiomyopathy/Schizophrenia and bipolar } \\
\text { disorder/Glioma/Breast cancer/Mitochondrial disease/Glaucoma/Leber } \\
\text { hereditary optic neuropathy/Lung cancer/GBM/Diabetes }\end{array}$ \\
\hline & $T 217 C$ & $\begin{array}{c}\text { Thyroid tumors/Chronic periodontitis/Schizophrenia and bipolar } \\
\text { disorder/Esophageal cancer/Diabetes/Mitochondrial disease/Leber } \\
\text { hereditary optic neuropathy/Epilepsy }\end{array}$ \\
\hline & C299A & None \\
\hline & A301C & None \\
\hline & A302insC & None \\
\hline & A302insCC & None \\
\hline & C308T & Diabetes type2/Glioma/ Noonan syndrome \\
\hline & Стзо9С & \\
\hline & T310C & $\begin{array}{c}\text { Melanoma/ Thyroid tumor/Renal oncocytoma/Alzheimer/Endometrial } \\
\text { carcinoma/Parotid carcinoma/Oncocytic pituitary }\end{array}$ \\
\hline
\end{tabular}


adenoma/Schizophrenia and bipolar

disorder/Cardiomyopathy/Epilepsy/Esophageal

cancer/GBM/Diabetes/Ovarian endometrioidserous Ovarian Cancer

$\begin{array}{cc}\text { T310ins C } & \text { None } \\ \text { T321C } & \text { Diabetes }\end{array}$

C340T Glioma/ Thyroid tumour/ Rhinopharinx ococytoma/ Schozoaffective disorder/ LHON/ GBM

C456T Thyroid tumour/Breast cancer/ Prostate cancer/ Renal oncocytoma/

Oncocytic pituitary adenoma/ Endometrial cancer type l/ Invasive mammary carcinoma/ Invasive primary mammary carcinoma/ Parotid carcinoma/ Serous ovarian cancer/ GBM/ Oral squamous cell carcinoma/ Parkinson's disease/ Diabetes type 2/ Obesity/ Chronic periodontitis/ Alzheimer's disease/ Schizophrenia/ Bipolar disorder type 1/ Noonan syndrome/ Mental disorder/ LHON/ Meningicaoccal disease

\section{None}

A508G
Thyroid tumor/Rhinopharinx oncocytoma/Chronic periodontitis/Schizophrenia and bipolar disorder/Esophageal cancer/Neuropathy/ Ataxia and retinitis pigmentosa (NARP)/ Fatal childhood maternally inherited Leigh's syndrome/Mitochondrial disease/Leber hereditary optic neuropathy/Diabetes
A567C

A567insC

A16051G

43

44

45

46

47

48

49

50

51

52

53

54

55

56

T16093C

C16111T

$\begin{array}{cc}\text { C510G } & \text { None } \\ \text { A512C } & \text { None } \\ \text { G513C } & \text { None } \\ \text { GCA513G } & \text { None } \\ \text { G513A } & \text { None } \\ \text { G513insCA } & \text { None } \\ \text { G564C } & \text { None } \\ \text { C566A } & \text { None }\end{array}$
None
None
None
None
None
None
None

\section{Ophthalmoplegia/ TWINKLE/PEO}

Thyroid tumor/Diabetes/Rhinopharinx oncocytoma/Chronic periodontitis/OXPHOS system deficiency/Schizophrenia and bipolar disorder/Noonan syndrome/Diabetes/Leber hereditary optic neuropathy/GBM

T16092C Leber hereditary optic neuropathy/Gastric cancer/Alzheimer's disease/Diabetes/Prostate cancer/Non-syndromic optic neuropathy

/Schizophrenia/Parkinson`s Disease/Esophageal cancer/Epilepsy/Meningococcal disease/GBM

None

Parkinson`s Disease/ Alzheimer's Disease/Diabetes/Obesity/Leber hereditary optic neuropathy/Schizophrenia/Prostate cancer

T16126C Glioblastoma/Thyroid tumor/Leber hereditary optic neuropathy/Renal oncocytoma/Parkinson`s Disease/ Alzheimer's Disease/Diabetes/Chronic 
G16129C

G16129A

G16153A

16179del2

CA16179C

A16181insC

A16182C

A16183C

A16183insC

16188del1

T16189C

C16192T
Progressive External Ophthalmoplegia/Oncocytic pituitary adenoma/Salivary mixed tumor/Tongue carcinoma/Warthin tumor/Chronic periodontitis/Prostate cancer/Endometrial carcinoma/Schizophrenia and bipolar disorder/Noonan syndrome/Breast cancer/Cardiomyopathy/Epilepsy/Non-syndromic optic neuropathy/Esophageal cancer/Glaucoma/Neurofibromatosis/Meningococcal disease/GBM/Ovarian cancer None None

Thyroid tumor/Leber hereditary optic neuropathy/Prostate cancer/Oncocytic pituitary adenoma/Esophageal cancer/Diabetes/GBM

None

None

None

Prostate tumour/ Myoclonic Epilepsy and Ragged Red Muscle Fibers/Parkinson`s Disease/ Alzheimer's

Disease/Diabetes/Schizophrenia/Breast cancer/Gastric cancer/Colorectal cancer/Leber hereditary optic neuropathy/MELAS/Schizophrenia and bipolar disorder/Noonan syndrome/Cardiomyopathy/Neurogenic muscle weakness/ Ataxia/ And Retinitis Pigmentosa/Maternally Inherited Syndrome/Cardiomyopathy/Esophageal cancer

\section{Lung tumour back-mutation/ Prostate tumour/ Thyroid tumor/Myoclonic} Epilepsy and Ragged Red Muscle Fibers/Parkinson`s Disease/ Alzheimer's

Disease/Diabetes/Breast cancer/Pituitary adenoma/Warthin

tumor/Gastric cancer/Chronic periodontitis/MELAS/Colorectal cancer/Prostate cancer/Leber hereditary optic neuropathy/Endometrial carcinoma/Prostate cancer/Schizophrenia and bipolar disorder/Hypertension/Noonan syndrome/Breast cancer/Cardiomyopathy/Neurogenic muscle weakness/Ataxia/ And Retinitis Pigmentosa/Maternally Inherited Syndrome/Esophageal cancer/Lung cancer/GBM/Ovarian cancer

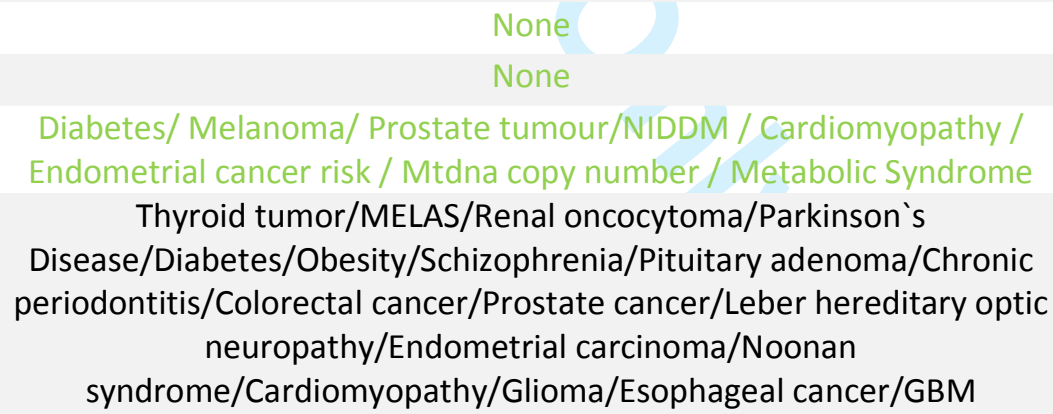
neuropathy/Endometrial carcinoma/Noonan syndrome/Cardiomyopathy/Glioma/Esophageal cancer/GBM

C16234T Thyroid tumor/Alzheimer's disease/Diabetes/Schizophrenia/Parkinson`s Disease/Obesity/Colorectal cancer/Prostate cancer/Endometrial carcinoma/Leber hereditary optic neuropathy/Esophageal cancer

C16256T Disease/Diabetes/Obesity/Gastric cancer/Prostate cancer/Alzheimer's 
disease/Endometrial carcinoma/Noonan syndrome/Breast cancer/Cardiomyopathy/Leber's hereditary optic neuropathy/Neurogenic muscle weakness/Ataxia/ And Retinitis Pigmentosa/Maternally Inherited

Syndrome/Esophageal cancer/Epilepsy/GBM/Meningococcal disease

A16258C

None

C16270T Thyroid tumor/MELAS/Renal oncocytoma/Colorectal cancer/Prostate cancer/Endometrial carcinoma/Pituitary adenoma/Glioma/Noonan syndrome/Cardiomyopathy/Leber hereditary optic

neuropathy/Diabetes/Mitochondrial encephalopathy with lactic acidosis and stroke-like episodes/Glaucoma/Epilepsy/GBM

C16291T

Thyroid tumor/Parkinson`s Disease/ Alzheimer's

Disease/Diabetes/Obesity/Schizophrenia/Gastric cancer/Leber hereditary optic neuropathy/Endometrial carcinoma/Epilepsy/Glioma/Esophageal

cancer/Neurofibromatosis/Lung cancer/Meningococcal disease/GBM/Serous Ovarian Cancer

C16294T

Thyroid tumor/Parkinson`s Disease/ Alzheimer's Disease/Diabetes/Obesity/Schizophrenia/Renal oncocytoma/Leber hereditary optic neuropathy/Pituitary adenoma/Tongue carcinoma/ Salivary mixed tumor/Warthin tumor/Prostate cancer/Endometrial carcinoma/Schizophrenia and bipolar disorder/Noonan syndrome/Cardiomyopathy/Breast cancer/Neurogenic muscle weakness/ Ataxia/ And Retinitis Pigmentosa/Maternally Inherited Syndrome/Glaucoma/Neurofibromatosis/Epilepsy/GBM/Serous Ovarian Cancer

C16296T Thyroid tumor/Renal oncocytoma/Leber hereditary optic neuropathy/Pituitary adenoma/Tongue carcinoma/ Salivary mixed tumor/Warthin tumor/Prostate cancer/Schizophrenia and bipolar

disorder/Noonan syndrome/Breast cancer/Diabetes/GBM

T16304C Esophageal/ Breast \& prostate tumours/Thyroid tumor/Parkinson`s Disease/ Alzheimer's Disease/Diabetes/Obesity/Schizophrenia/Renal oncocytoma/Leber hereditary optic neuropathy/Pituitary adenoma/Parotid carcinoma/Warthin tumor/Gastric cancer/Chronic periodontitis/Colorectal cancer/Prostate cancer/Endometrial carcinoma/Esophageal cancer/Maternally inherited essential hypertension/Noonan syndrome/Periodic paralyses and neuropathy/Lung cancer/Meningococcal disease/Epilepsy/GBM/Serous Ovarian Cancer

T16311C

A16316G

C16320T

\section{Prostate tumour}

Parkinson`s Disease/ Alzheimer's Disease/Diabetes/Obesity/Leber hereditary optic neuropathy/Schizophrenia/Esophageal cancer/Epilepsy/Oral squamous cell carcinoma

Thyroid tumor/Renal oncocytoma/Chronic periodontitis/Diabetes/Obesity/Alzheimer's disease/Prostate cancer/Warthin tumor/Schizophrenia and bipolar disorder/Leber Hereditary Optic Neuropathy/OXPHOS system deficiency/Glaucoma/Meningococcal disease/Lung cancer 
T16362C Thyroid tumor/Leber hereditary optic neuropathy/MELAS/Parkinson`s Disease/ Alzheimer's Disease/Diabetes/Obesity/Rhinopharinx oncocytoma/Gastric cancer/Pituitary adenoma/Warthin tumor/Chronic periodontitis/Colorectal cancer/Prostate cancer/Ovarian cancer/Endometrial carcinoma/Schizophrenia and bipolar disorder/Hypertension/Noonan syndrome/Breast cancer/Cardiomyopathy/Neurogenic muscle weakness/ Ataxia/ And Retinitis Pigmentosa/Maternally Inherited Syndrome/Esophageal cancer/Glaucoma/Meningococcal disease/Lung cancer/Epilepsy/Oral squamous cell carcinoma/GBM/Serous Ovarian Cancer

A16399G

Gastric carcinoma/ Thyroid tumor/MELAS/Parkinson`s Disease/ Alzheimer's Disease/Diabetes/Obesity/Renal oncocytoma/Endometrial carcinoma/Prostate cancer/Noonan

syndrome/Cardiomyopathy/Oncocytic pituitary adenoma/Leber hereditary optic neuropathy/Esophageal

cancer/Glaucoma/Schizophrenia/Epilepsy/GBM/Non-syndromic Hearing loss/Oral squamous cell carcinoma

T16519C Glioblastoma/ Gastric/Lung/ Ovarian/ Prostate tumours

G16526A Thyroid tumor/Prostate cancer/Leber hereditary optic neuropathy/Cardiomyopathy/Diabetes/Epilepsy/GBM

\begin{tabular}{|c|c|c|}
\hline \multirow[t]{3}{*}{ Mt-rnr1 } & G709A & None \\
\hline & G930A & $0>0$ \\
\hline & G988A & Possible DEAF risk factor \\
\hline \multirow[t]{11}{*}{ Mt-rnr2 } & A1811G & Head/Neck tumour \\
\hline & G1888A & None \\
\hline & A2581G & $\begin{array}{l}\text { Thyroid tumor/Prostate cancer/Leber hereditary optic } \\
\text { neuropathy/OXPHOS system deficiency/Noonan syndrome/Diabetes }\end{array}$ \\
\hline & A2706G & None \\
\hline & G3010A & $\begin{array}{c}\text { Cyclic Vomiting Syndrome with Migraine/Thyroid tumor/Leber } \\
\text { hereditary optic neuropathy/Renal } \\
\text { oncocytoma/Obesity/Diabetes/Colorectal cancer/Prostate } \\
\text { cancer/Pituitary adenoma/Parotid carcinoma/Endometrial } \\
\text { carcinoma/OXPHOS system deficiency/Subcortical infarcts and } \\
\text { leukoencephalopathy/Alivary benign neoplasia/Schizophrenia and } \\
\text { bipolar disorder/Noonan syndrome/Cardiomyopathy/Breast cancer/Non- } \\
\text { syndromic optic neuropathy /Esophageal } \\
\text { cancer/Diabetes/Glaucoma/Periodic paralyses and } \\
\text { neuropathy/Neurofibromatosis/Meningococcal disease/Lung } \\
\text { cancer/Epilepsy/GBM/Ovarian endometrioid/Serous Ovarian Cancer }\end{array}$ \\
\hline & AC3105A & None \\
\hline & A3105T & None \\
\hline & T3197C & None \\
\hline & G3226T & None \\
\hline & T3229A & None \\
\hline & T3229insA & None \\
\hline
\end{tabular}


Thyroid tumor/Chronic periodontitis/Parkinson`s Disease/Rhinopharinx oncocytoma/Schizophrenia and bipolar disorder/OXPHOS system deficiency/Leber hereditary optic neuropathy/Neurogenic muscle weakness/ Ataxia/ And Retinitis Pigmentosa/Maternally Inherited Syndrome/GBM/Diabetes

A3796G

Adult-onset dystonia/ Prostate cancer/OXPHOS deficiency/Subcortical (T164A)

G3849A infarcts and leukoencephalopathy (CADASIL)/Schizophrenia and bipolar disorder/Diabetes

Chronic periodontitis/OXPHOS deficiency/Noonan syndrome/Esophageal cancer/Diabetes

Thyroid tumour/PD/Thyroid tumour/Renal oncocytoma/Parotid oncocytoma/Endometrial carcinoma/Pituitary adenoma/OXPHOS deficiency/Noonan syndrome /Parotid carcinoma/Diabetes/GBM

PD/Thyroid tumor/Renal oncocytoma/Parotid oncocytoma/Endometrial carcinoma/Pituitary adenoma/OXPHOS deficiency/Noonan syndrome /Parotid carcinoma/Diabetes/GBM 
A5390G

(M307M)

G5147A

(T226T)

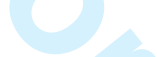

T5426C

( $\mathrm{H} 319 \mathrm{H})$ oncocytoma/Diabetes/Pituitary adenoma/Parotid carcinoma/OXPHOS system deficiency/Noonan syndrome/Endometrial cancer

Thyroid tumor/Renal oncocytoma/Obesity/Gastric cancer/ Oncocytic pituitary adenoma/Leber hereditary optic neuropathy/Endometrial carcinoma/OXPHOS system deficiency/Salivary mixed tumor/Warthin tumor/Parotid oncocytoma/Epilepsy/Noonan syndrome/Breast cancer/Schizophrenia/Esophageal cancer/Glaucoma/Lung cancer/GBM/Diabetes

Thyroid tumor/Rhinopharinx oncocytoma/Chronic periodontitis/OXPHOS system deficiency/Subcortical infarcts and leukoencephalopathy/Schizophrenia and bipolar disorder/Neurogenic muscle weakness/ Ataxia/ And Retinitis Pigmentosa/Maternally Inherited Syndrome/Diabetes/Leber hereditary optic neuropathy

\section{Thyroid tumor/Rhinopharinx oncocytoma/Chronic periodontitis/OXPHOS} system deficiency/Subcortical infarcts and leukoencephalopathy/Schizophrenia and bipolar disorder/Neurogenic muscle weakness/ Ataxia/ And Retinitis Pigmentosa/Maternally Inherited Syndrome /Esophageal cancer/Diabetes/Leber hereditary optic neuropathy/Epilepsy/GBM

\section{Mt-nd4}

\section{A10876G \\ (L39L)}

A11251G

(L164L)

T11353C (A198A)

A11467G (L236L)
Thyroid tumor/Rhinopharinx oncocytoma/Chronic periodontitis/OXPHOS system deficiency/Subcortical infarcts and leukoencephalopathy/Schizophrenia and bipolar disorder/Cardiomyopathy/Esophageal cancer/Diabetes/Leber hereditary optic neuropathy/GBM

Thyroid tumor/Leber hereditary optic neuropathy/Renal oncocytoma/Chronic Progressive External Ophthalmoplegia/Pituitary adenoma/Tongue carcinoma/Salivary mixed tumor/Warthin tumor/Parotid oncocytoma/Endometrial carcinoma/Prostate cancer/OXPHOS system deficiency/Subcortical infarcts and leukoencephalopathy/Schizophrenia and bipolar disorder/Noonan syndrome/Breast cancer/Cardiomyopathy/Epilepsy/Non-syndromic optic neuropathy/Diabetes/Glaucoma/Neurofibromatosis/Serous Ovarian Cancer/GBM GBM/Cardiomyopathy/Leber's hereditary optic neuropathy Altered brain ph/Thyroid tumor/MELAS/Renal oncocytoma/Leber hereditary optic neuropathy/Rhinopharinx oncocytoma/Pituitary adenoma/Oral cavity carcinoma/Warthin tumor/Chronic periodontitis/Prostate cancer/Endometrial carcinoma/OXPHOS system deficiency/Subcortical infarcts and leukoencephalopathy/Schizophrenia and bipolar disorder/Gliomas/Noonan syndrome/Breast cancer/Cardiomyopathy/Non-syndromic hearing loss/Autosomal dominant opticatrophy/Neurogenic muscle weakness/Ataxia/ And Retinitis Pigmentosa/Maternally Inherited 
Syndrome/Diabetes/Neurofibromatosis/Epilepsy/GBM/Serous Ovarian Cancer

G11719A

None

(G320G)

A11812G Thyroid tumor/Renal oncocytoma/ Leber hereditary optic (L351L) neuropathy/Oncocytic pituitary adenoma/Salivary mixed tumor/Tongue carcinoma/Warthin tumor/Prostate cancer/Endometrial carcinoma/OXPHOS system deficiency/Schizophrenia and bipolar disorder/Noonan syndrome/Cardiomyopathy/Epilepsy/Breast cancer/Diabetes/GBM/Oral squamous cell carcinoma/Serous Ovarian Cancer

\begin{tabular}{|c|c|c|}
\hline$M t-n d 4 L$ & $\begin{array}{c}\text { C10619T } \\
\text { (N50N) }\end{array}$ & Thyroid tumor/Diabetes/Oral squamous cell carcinoma \\
\hline \multirow[t]{4}{*}{ Mt-nd5 } & $\begin{array}{c}\text { G12372A } \\
\text { (L12L) }\end{array}$ & $\begin{array}{c}\text { Altered brain ph/Prostate tumour/Thyroid cancer/Breast } \\
\text { cancer/MELAS/Obesity/Renal oncocytoma/Leber Hereditary } \\
\text { Optic/Chronic Periodontitis Neuropathy/Warthin tumor/Colorectal } \\
\text { cancer/Endometrial cancer type I/OXPHOS system deficiency/Cerebral } \\
\text { arteriopathy with subcortical infarcts and } \\
\text { leukoencephalopathy/Schizophrenia/Neurofibromatosis type 1/Leber } \\
\text { Hereditary Optic Neuropathy/Noonan syndrome/Autosomal dominant } \\
\text { opticatrophy/Diabetes/Periodic paralyses and neuropathy/Hearing } \\
\text { loss/Meningococcal disease/Serous Ovarian Cancer/Epilepsy }\end{array}$ \\
\hline & $\begin{array}{c}\text { A12397G } \\
\text { (T21A) }\end{array}$ & $\begin{array}{c}\text { Early-onset PD/Thyroid tumor/Endometrial } \\
\text { carcinoma/GBM/Diabetes/OXPHOS system deficiency/Parkinson`s } \\
\text { Disease/Tongue carcinoma }\end{array}$ \\
\hline & $\begin{array}{l}\text { C12557T } \\
\text { (T74I) }\end{array}$ & $\begin{array}{l}\text { Chronic periodontitis/Pituitary adenoma/OXPHOS system } \\
\text { deficiency/Glaucoma/Leber hereditary optic neuropathy/Diabetes }\end{array}$ \\
\hline & $\begin{array}{l}\text { T13020C } \\
\text { (G228G) }\end{array}$ & $\begin{array}{c}\text { Thyroid tumor/Rhinopharinx oncocytoma/Chronic periodontitis/OXPHOS } \\
\text { system deficiency/Subcortical infarcts and } \\
\text { leukoencephalopathy/Schizophrenia and bipolar disorder/Neurogenic } \\
\text { muscle weakness/ Ataxia/ And Retinitis Pigmentosa/Maternally Inherited } \\
\text { Syndrome/Diabetes/Leber hereditary optic neuropathy/GBM }\end{array}$ \\
\hline
\end{tabular}

G13368A

(G344G)

Thyroid tumor/Leber hereditary optic neuropathy/Renal clear cell carcinoma/Chronic periodontitis/Prostate cancer/Endometrial carcinoma/OXPHOS system deficiency/Pituitary adenoma/Tongue carcinoma/Salivary mixed tumor/Warthin tumor/Parotid oncocytoma/Subcortical infarcts and leukoencephalopathy/Schizophrenia and bipolar disorder/Glioma/Noonan syndrome/Cardiomyopathy/Epilepsy/Breast cancer/Diabetes/Neurofibromatosis/GBM/Serous Ovarian Cancer

A13419T Non-syndromic Hearing loss

(G361G)

T13617C (1427I)

Thyroid tumor/MELAS/Renal oncocytoma/ Diabetes/Pituitary adenoma/Prostate cancer/Endometrial carcinoma/OXPHOS system 
deficiency/Subcortical infarcts and leukoencephalopathy/Glioma/Noonan syndrome/Cardiomyopathy/Leber's hereditary optic neuropathy/Diabetes/Epilepsy/GBM/ Neurogenic muscle weakness/ Ataxia and retinitis pigmentosa/ Maternally inherited form of Leigh syndrome

T13734C Thyroid tumor/Renal oncocytoma/Chronic periodontitis/OXPHOS system (F466F) deficiency/Rhinopharinx oncocytoma/Subcortical infarcts and leukoencephalopathy/Schizophrenia and bipolar disorder/Diabetes/Leber hereditary optic neuropathy/GBM

G13813A None

(V493I)

G13889A

(C518Y)

Head/Neck tumour/Parotid oncocytoma/Schizophrenia/Mitochondrial disease

A14145G

Prostate cancer

\begin{tabular}{|c|c|c|}
\hline \multirow[t]{3}{*}{$M t-n d 6$} & $\begin{array}{c}\text { A14233G } \\
\text { (D147D) }\end{array}$ & $\begin{array}{c}\text { Thyroid tumor/Leber hereditary optic neuropathy/Prostate } \\
\text { cancer/Endometrial carcinoma/Oncocytic pituitary adenoma/OXPHOS } \\
\text { system deficiency/Schizophrenia and bipolar disorder/Tongue } \\
\text { carcinoma/Noonan syndrome/Cardiomyopathy/Salivary mixed } \\
\text { tumor/Breast cancer/Warthin tumor/Epilepsy/Esophageal } \\
\text { cancer/Diabetes/GBM/Oral squamous cell carcinoma/Serous Ovarian } \\
\text { Cancer }\end{array}$ \\
\hline & $\begin{array}{l}\text { C14365T } \\
\text { (V103V) }\end{array}$ & $\begin{array}{c}\text { Thyroid tumor/Renal oncocytoma/Endometrial carcinoma/OXPHOS } \\
\text { system deficiency/Pituitary adenoma/Parotid } \\
\text { carcinoma/Diabetes/Noonan syndrome }\end{array}$ \\
\hline & $\begin{array}{c}\text { A14582G } \\
\text { (V31A) }\end{array}$ & $\begin{array}{l}\text { Thyroid tumor/Renal oncocytoma/Endometrial carcinoma/Parkinson`s } \\
\text { Disease/Pituitary adenoma/Parotid carcinoma/OXPHOS system } \\
\text { deficiency/Noonan syndrome/Diabetes/GBM/ Ovarian endometrioid }\end{array}$ \\
\hline \multirow[t]{2}{*}{$M t-c y b$} & $\begin{array}{c}C 14766 T \\
\text { (T7I) }\end{array}$ & None \\
\hline & $\begin{array}{c}\text { A14793G } \\
\text { (H16R) }\end{array}$ & $\begin{array}{c}\text { Thyroid tumor/Renal oncocytoma/MELAS/Prostate cancer/Endometrial } \\
\text { carcinoma/Alzheimer's Disease/OXPHOS system deficiency/Subcortical } \\
\text { infarcts and leukoencephalopathy/Noonan } \\
\text { syndrome/Cardiomyopathy/Leber hereditary optic } \\
\text { neuropathy/Diabetes/GBM/Serous Ovarian Cancer }\end{array}$ \\
\hline
\end{tabular}

T14819insTCTAT $A$ G14905A (M53M)
None

Thyroid tumor/Renal oncocytoma/Leber hereditary optic neuropathy/Oncocytic pituitary adenoma/Salivary mixed tumor/ Warthin tumor/Gastric cancer/Prostate cancer/Endometrial carcinoma/OXPHOS system deficiency/Subcortical infarcts and leukoencephalopathy/Schizophrenia and bipolar disorder/Noonan 
syndrome/Cardiomyopathy/Epilepsy/Breast cancer/Diabetes/Glaucoma/Neurofibromatosis/GBM

A15218G (T158A) (L236I)

T15461C (L239L)

A15607G (K287K)
Thyroid tumor/Renal oncocytoma/MELAS/Leber hereditary optic neuropathy/Diabetes/Endometrial carcinoma/Prostate cancer/OXPHOS system deficiency/Noonan syndrome/Pituitary adenoma/Cardiomyopathy/Esophageal cancer/Neurogenic muscle weakness/Ataxia/ And Retinitis Pigmentosa/Maternally Inherited Syndrome/Lung cancer/GBM

Thyroid tumor/Leber hereditary optic neuropathy/Renal oncocytoma/Chronic Progressive External Ophthalmoplegia/Pituitary adenoma/Oncocytic pituitary adenoma/Salivary mixed tumor/Tongue carcinoma/Warthin tumor/Prostate cancer/Endometrial carcinoma/OXPHOS system deficiency/Subcortical infarcts and leukoencephalopathy/Schizophrenia and bipolar disorder/Noonan syndrome/Cardiomyopathy/Epilepsy/Breast cancer/Diabetes/Glaucoma/Neurofibromatosis/GBM/Ovarian cancer 
bipolar disorder/Glioma/Noonan syndrome/Breast cancer/Cardiomyopathy/Diabetes/Epilepsy/GBM/Serous Ovarian Cancer

\begin{tabular}{|c|c|c|}
\hline & $\begin{array}{l}\text { C7028T } \\
\text { (A375A) }\end{array}$ & None \\
\hline \multirow[t]{7}{*}{ Mt-co2 } & $\begin{array}{l}\text { T8265C } \\
\text { (L227P) }\end{array}$ & None \\
\hline & $\begin{array}{l}\text { A8266C } \\
\text { (L227L) }\end{array}$ & None \\
\hline & $\begin{array}{c}T 8267 C \\
(\mathrm{STOP}>\mathrm{Q})\end{array}$ & None \\
\hline & 8267delAGC & None \\
\hline & $\begin{array}{c}A 8268 T \\
(\mathrm{STOP}>\mathrm{L})\end{array}$ & None \\
\hline & $\begin{array}{c}\text { G8269A } \\
\text { (STOP }>S T O P)\end{array}$ & $\begin{array}{c}\text { Thyroid tumor/Renal oncocytoma/Pituitary adenoma/Parotid } \\
\text { oncocytoma/Colorectal cancer/OXPHOS system deficiency/Noonan } \\
\text { syndrome/Diabetes/GBM/ Ovarian cancer }\end{array}$ \\
\hline & $\begin{array}{c}G 8269 C \\
(S T O P>Y)\end{array}$ & None \\
\hline \multirow[t]{2}{*}{$M t-c o 3$} & $\begin{array}{l}\text { C9469T } \\
\text { (T88I) }\end{array}$ & Diabetes \\
\hline & $\begin{array}{c}\text { G9477A } \\
\text { (V91I) }\end{array}$ & $\begin{array}{l}\text { Thyroid tumour/Thyroid tumour/MELAS/Renal oncocytoma/Alzheimer's } \\
\text { Disease/Parkinson`s Disease/Pituitary adenoma/Prostate } \\
\text { cancer/Endometrial carcinoma/OXPHOS system deficiency/Subcortical } \\
\text { infarcts and leukoencephalopathy/Noonan } \\
\text { syndrome/Glioma/Cardiomyopathy/Diabetes/Epilepsy/GBM }\end{array}$ \\
\hline
\end{tabular}

A9664G

Diabetes

(E153G)

A9667G

(N154S)

Thyroid tumor/ MELAS/Prostate cancer/Leber's hereditary optic neuropathy/Parkinson`s Disease/Diabetes/Epilepsy

T9845C

(T213T)

None

C8622A

(P32P)

G8697A Thyroid tumour/Thyroid tumour/Renal oncocytoma/Leber hereditary

(M57M) optic neuropathy/Oncocytic pituitary adenoma/Tongue carcinoma/Salivary mixed tumor/Warthin tumor/Parotid oncocytoma/Prostate cancer/Endometrial carcinoma/OXPHOS system deficiency/Subcortical infarcts and leukoencephalopathy/Schizophrenia 
and bipolar disorder/Noonan

syndrome/Cardiomyopathy/Epilepsy/Breast

cancer/Diabetes/Neurofibromatosis/Leber hereditary optic neuropathy/GBM

T8821C

None

(S99P)

G8989A

(A155T)

G8994A

(L156L)

Thyroid tumor/Renal oncocytoma/Parkinson`s Disease/Alzheimer's

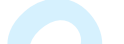

Disease/Chronic periodontitis/Oncocytic pituitary adenoma/Warthin

tumor/Prostate cancer/OXPHOS system deficiency/Subcortical infarcts and leukoencephalopathy/Diabetes/Breast cancer/Epilepsy

G9123A

Thyroid tumor/Leber hereditary optic neuropathy/Renal

(L199L) oncocytoma/Alzheimer's Disease/Parkinson`s Disease/Pituitary adenoma/Parotid carcinoma/Gastric cancer/Endometrial carcinoma/OXPHOS system deficiency/Noonan syndrome/Esophageal cancer/Diabetes

\begin{tabular}{|c|c|c|}
\hline Mt-atp8 & $\begin{array}{l}\text { T8473C } \\
\text { (P36P) }\end{array}$ & $\begin{array}{c}\text { Chronic periodontitis/Obesity/Diabetes/Prostate cancer/Pituitary } \\
\text { adenoma/OXPHOS system deficiency/Schizophrenia and bipolar } \\
\text { disorder/Cardiomyopathy/Leber hereditary optic neuropathy/Esophageal } \\
\text { cancer }\end{array}$ \\
\hline$M t-t q$ & T4336C & $\begin{array}{l}\text { AD/PD/ Hearing loss \& migraine/ Breast cancer/ Glioma/ Oncocytic } \\
\text { pituitary adenoma/ Endometrial cancer type I/ Alzheimer's disease/ } \\
\text { Noonan syndrome/ Meningicoccal disease }\end{array}$ \\
\hline Mt-tm & A4435G & $\begin{array}{c}\text { LHON modulator / Hypertension/ Leber hereditary optic } \\
\text { neuropathy/Diabetes }\end{array}$ \\
\hline$M t-t r$ & T10463C & $\begin{array}{c}\text { Endometrium tumour/ Thyroid tumor/Renal oncocytoma/Leber } \\
\text { hereditary optic neuropathy/Oncocytic pituitary adenoma/Tongue } \\
\text { carcinoma/Salivary mixed tumor/Warthin tumor/Parotid } \\
\text { oncocytoma/Prostate cancer/Endometrial carcinoma/OXPHOS system } \\
\text { deficiency/Subcortical infarcts and leukoencephalopathy/Schizophrenia } \\
\text { and bipolar disorder/Noonan } \\
\text { syndrome/Cardiomyopathy/Epilepsy/Breast } \\
\text { cancer/Diabetes/Neurofibromatosis/Gbmserous Ovarian Cancer }\end{array}$ \\
\hline$M t-t / 2$ & A12308G & $\begin{array}{l}\text { CPEO / Stroke / Cardiomyopathy / Breast \& Renal \& Prostate Cancer Risk } \\
\text { / Altered brain ph/Lung \& prostate tumour/ Thyroid cancer/Breast } \\
\text { cancer/Serous Ovarian Cancer/Glioma/MELAS/Renal oncocytoma/Leber } \\
\text { Hereditary Optic Neuropathy /Pituitary adenoma/Oral cavity carcinoma } \\
\text { /Warthin tumor/Chronic Periodontitis/Prostate cancer/Endometrial } \\
\text { cancer type I/Cerebral arteriopathy with subcortical infarcts and } \\
\text { leukoencephalopathy/Schizoaffective disorder/Neurofibromatosis type } \\
\text { 1/Noonan syndrome/Meningococcal disease/Epilepsy /Diabetes }\end{array}$ \\
\hline$M t-t t$ & A15907G & $\begin{array}{c}\text { Thyroid tumor/Chronic periodontitis/OXPHOS system } \\
\text { deficiency/Rhinopharinx oncocytoma/Subcortical infarcts and } \\
\text { leukoencephalopathy/Schizophrenia and bipolar }\end{array}$ \\
\hline
\end{tabular}

URL: http://mc.manuscriptcentral.com/gdna Email: desalle@amnh.org 
disorder/Diabetes/Leber hereditary optic neuropathy/Neurogenic muscle weakness, ataxia and Retinitis Pigmentosa/Maternally Inherited Syndrome

G15928A Multiple Sclerosis / Idiopathic repeat miscarriage / AD protection/ Thyroid tumor/Renal oncocytoma/Diabetes/Leber hereditary optic neuropathy/Endometrial carcinoma/Prostate cancer/OXPHOS system deficiency/Subcortical infarcts and leukoencephalopathy/Schizophrenia and bipolar disorder/Noonan syndrome/Cardiomyopathy/Pituitary adenoma/Tongue carcinoma/Salivary mixed tumor/Warthin tumor/Chronic periodontitis/Glioma/Epilepsy/Breast cancer/Esophageal cancer/Neurofibromatosis/GBM/Serous Ovarian Cancer

\begin{tabular}{c|cc}
\hline Mt-olr & G5746A & Chronic periodontitis/Diabetes/Leber hereditary optic neuropathy \\
\hline $\begin{array}{c}\text { Other non- } \\
\text { coding } \\
\text { regions }\end{array}$ & T5892A & None \\
& & \\
& A5894C & Gastric carcinoma \\
& A5894CC & None \\
& C8270T & Gastric cancer/Diabetes/Oncocytic pituitary adenoma/Mitochondrial \\
(NC7) & disease/Epilepsy \\
& N8270insACCCC & None \\
& CTCT & None \\
& T8277insTCTAC & \\
& CCCCC &
\end{tabular}

Abbreviations: AD - Alzheimer's disease/ PD - Parkinson's disease/ Sporadic progressive external ophthalmoplegia/ DEAF - Maternally inherited deafness or aminoglycoside-induced deafness/ NTRIPN - Antiretroviral Therapy-Associated Peripheral Neuropathy/ MELAS - Mitochondrial Encephalomyopathy Lactic Acidosis and Stroke like episodes/ CPEO - Chronic Progressive External Ophthalmoplegia. 
Figure S1. Location of non-synonymous amino acid substitution sites following the alignments of human ( $\mathrm{CCRS}$ ) against the bovine homologous mtDNA-encoded polypeptide sequences. The human amino acid substitutions are shown in the boxes first, followed by the amino acid that is present in the bovine model in brackets.

\section{Complex I}

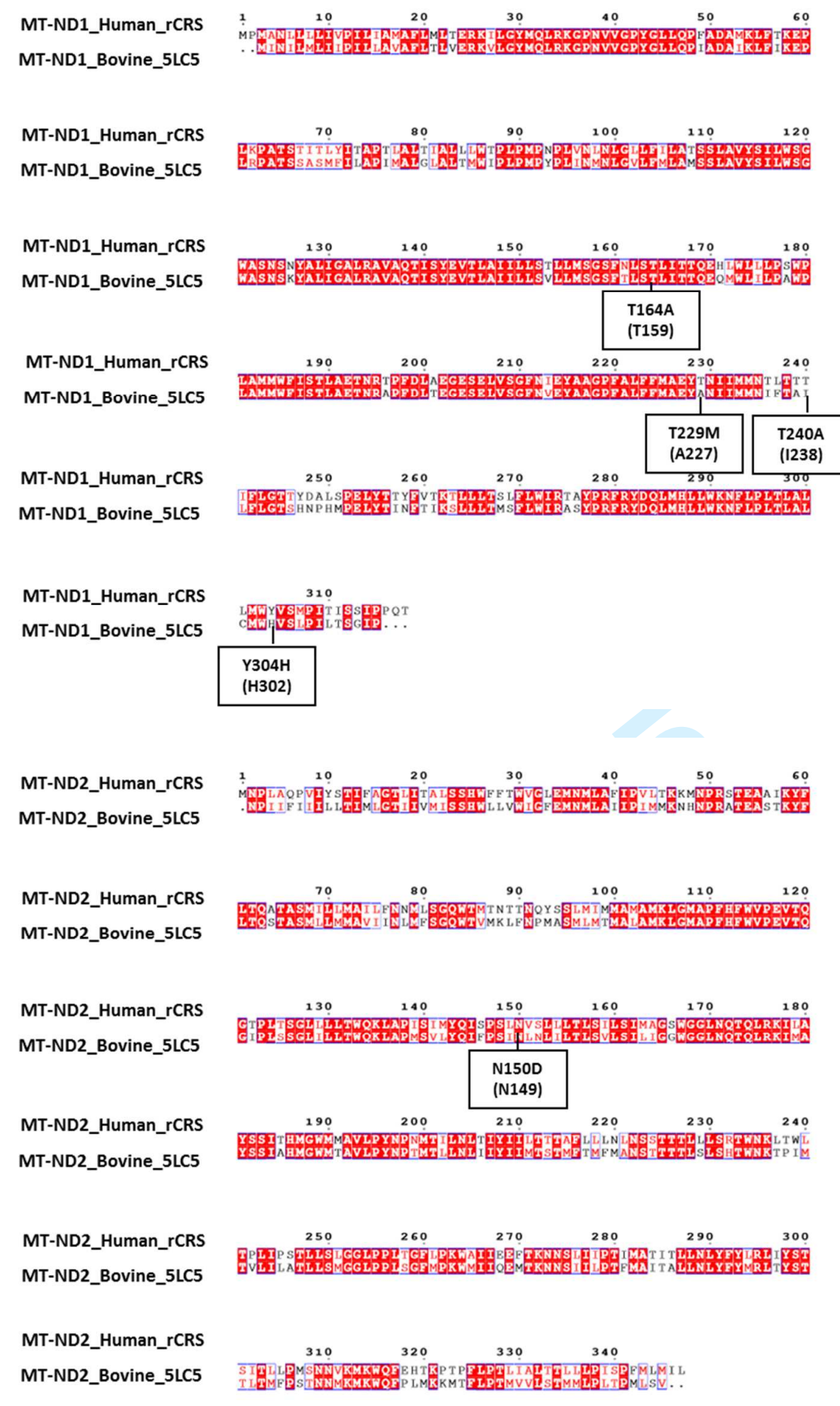

URL: http://mc.manuscriptcentral.com/gdna Email: desalle@amnh.org 
MT-ND5_Human_rCRS

MT-ND5_Bovine_5LC5

MT-ND5_Human_rCRS

MT-ND5_Bovine_5LC5

MT-ND5_Human_rCRS

MT-ND5_Bovine_5LC5

MT-ND5_Human_rCRS

MT-ND5_Bovine_5LC5

MT-ND5_Human_rCRS

MT-ND5_Bovine_5LC5

MT-ND5_Human_rCRS

MT-ND5_Bovine_5LC5

MT-ND5_Human_rCRS

MT-ND5_Bovine_5LC5

MT-ND5_Human_rCRS

MT-ND5_Bovine_5LC5

MT-ND5_Human_rCRS

MT-ND5_Bovine_5LC5

MT-ND5_Human_rCRS

MT-ND5_Bovine_5LC5

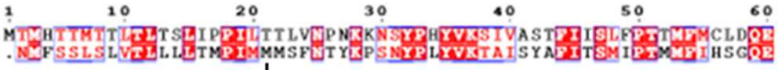

\section{T21A}

$70 \quad 90 \quad 100 \quad 110 \quad 120$

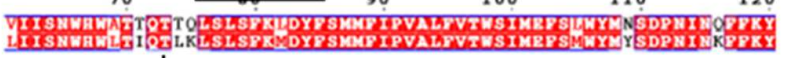

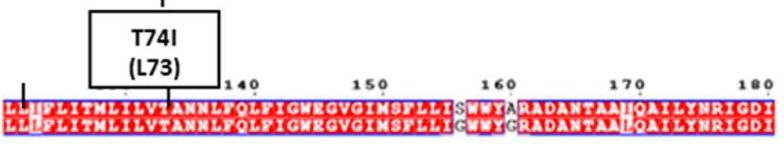

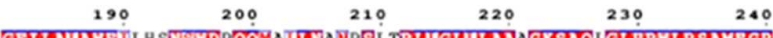

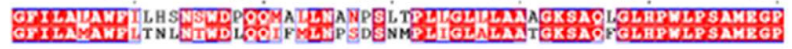

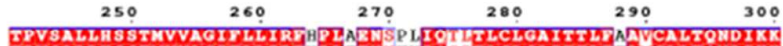

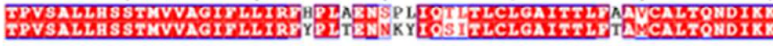

\section{G}

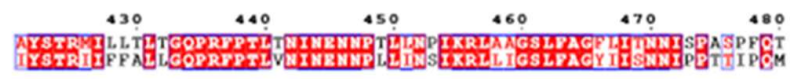

$\begin{array}{llllll}490 & 500 & 510 & 520 & 530 & 540\end{array}$

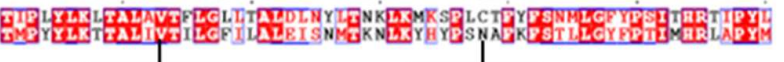

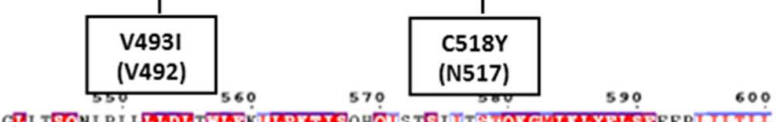

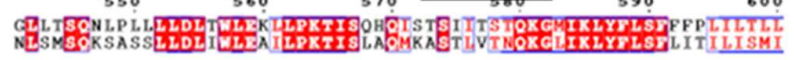

MT-ND5_Human_rCRS

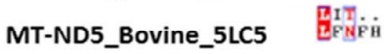

MT-ND6_Human_rCRS

MT-ND6_Bovine_5LC5

MT-ND6_Human_rCRS MT-ND6_Bovine_5LC5

MT-ND6_Human_rCRS MT-ND6_Bovine_5LC5

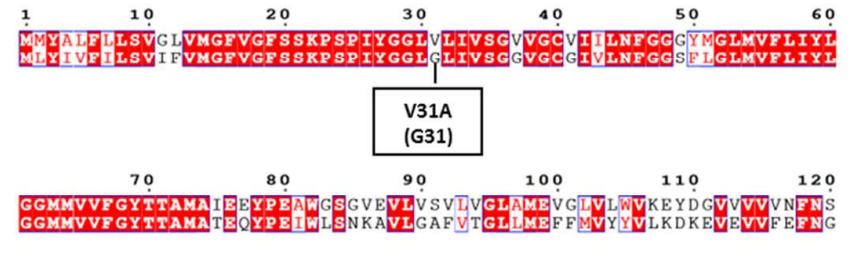

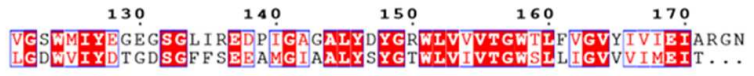




\section{Complex III}

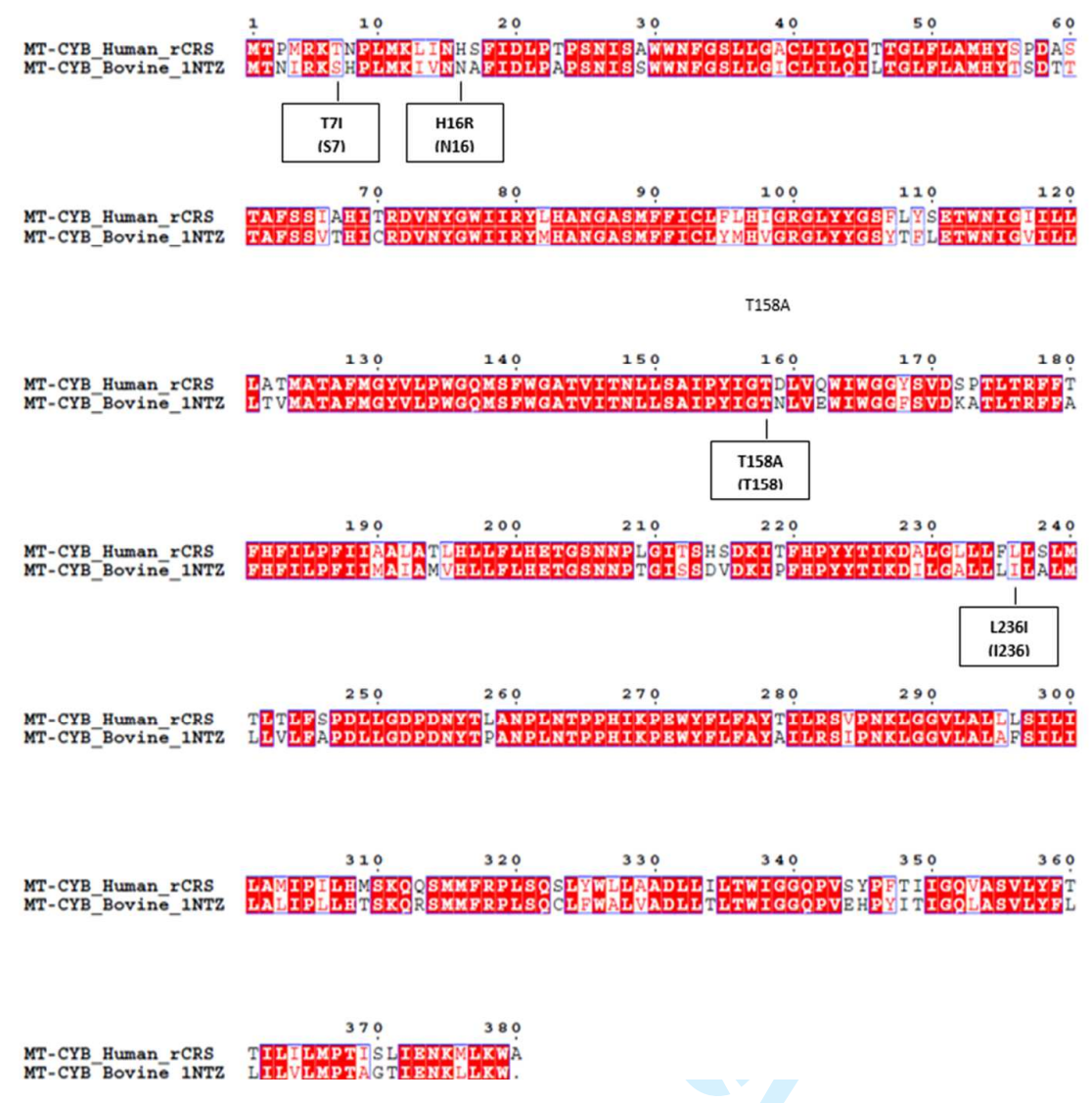

MT-CYB Buman_rCRS
MT-CYB Bovine
1NTZ

URL: http://mc.manuscriptcentral.com/gdna Email: desalle@amnh.org 


\section{Complex IV}
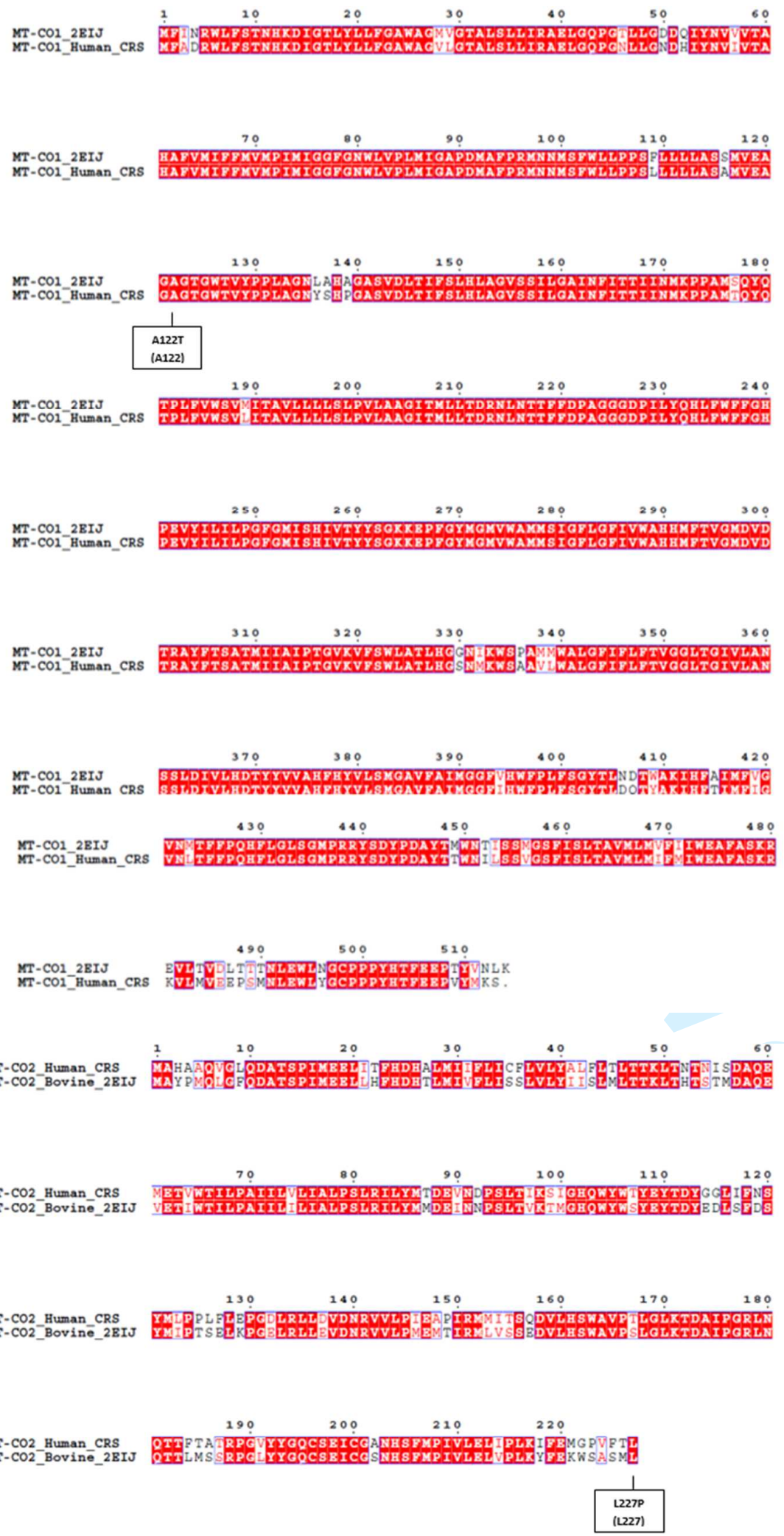

URL: http://mc.manuscriptcentral.com/gdna Email: desalle@amnh.org 


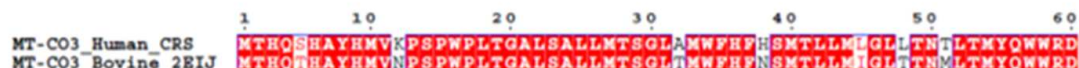

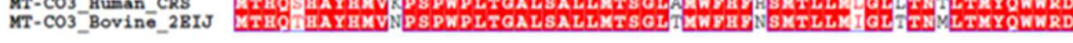

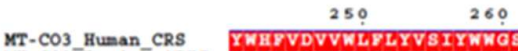

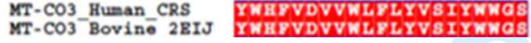

\section{Complex V}

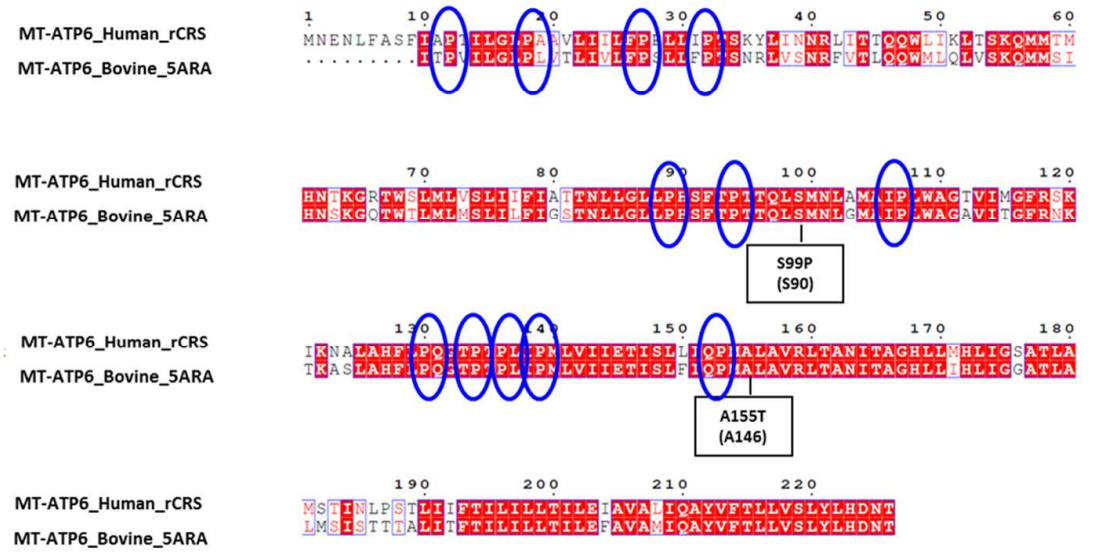

Conserved prolines that relate to Figure 7 in the main text are highlighted in blue.

URL: http://mc.manuscriptcentral.com/gdna Email: desalle@amnh.org 
Figure S2. Comparison of the wild type $m t-c y b$ and MT-CYB sequences against the variant sequences which contain a 6-nuclotide insertion at position 14819.

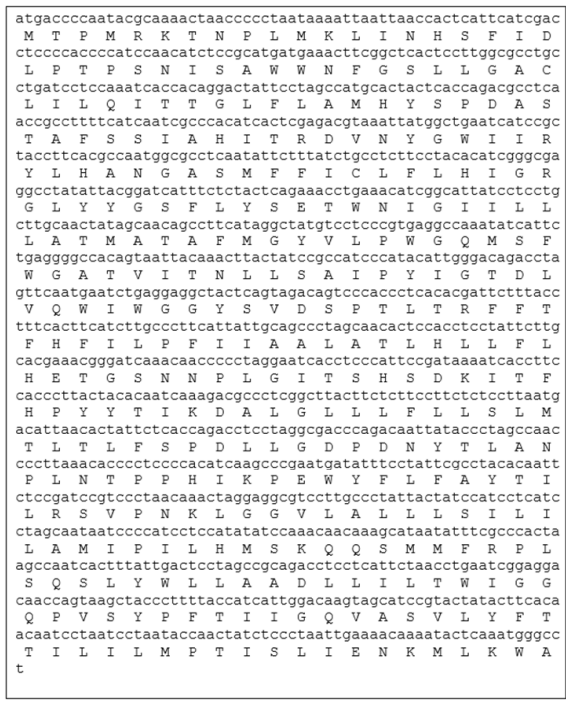

Wild type

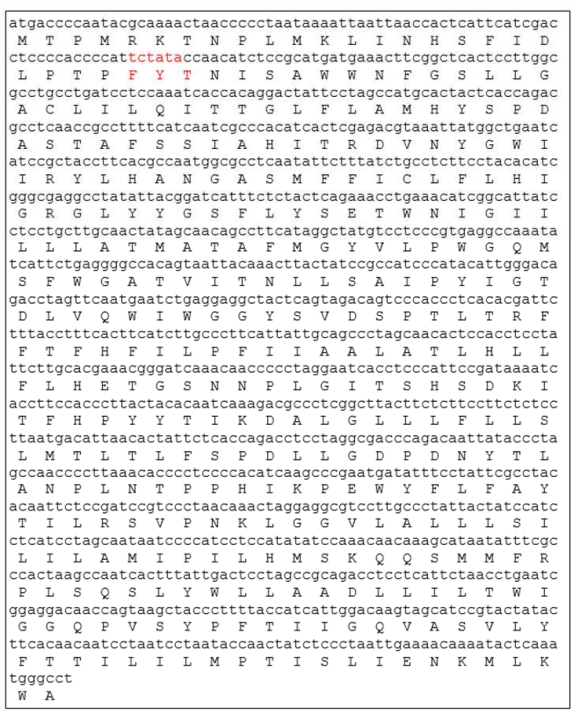

T14819insTCTATA Variant Sequence

URL: http://mc.manuscriptcentral.com/gdna Email: desalle@amnh.org 


\section{References}

1. Lloyd, R.E.; McGeehan, J.E. Structural Analysis of Mitochondrial Mutations Reveals a Role for Bigenomic Protein Interactions in Human Disease. PLoS One. 2013, 8, e69003. 